\title{
Molecular and Crystalline Requirements for Solid State Fluorescence Exploiting Excited State Intramolecular Proton Transfer
}

\author{
Michael Dommett, Miguel Rivera, Matthew T. H. Smith, Rachel Crespo Otero
}

Submitted date: 17/06/2019 - Posted date: 18/06/2019

Licence: CC BY-NC-ND 4.0

Citation information: Dommett, Michael; Rivera, Miguel; Smith, Matthew T. H.; Crespo Otero, Rachel (2019): Molecular and Crystalline Requirements for Solid State Fluorescence Exploiting Excited State Intramolecular Proton Transfer. ChemRxiv. Preprint.

Aggregation induced emission offers a route to the development of emissive technologies based on solely organic systems. However, maximising fluorescence quantum efficiencies (QE) is a formidable challenge in attaining first-principles materials design, due to the interplay between the electronic structure of the chromophore and the molecular crystal. The identification of radiative and nonradiative channels, and how these are affected by aggregation, can rationalise the emissive properties of materials and aid in the design of yet more efficient fluorophores. In the current work, we examine the mechanism behind aggregation induced emission in two related families of compounds with lasing properties, which undergo excited state intramolecular proton transfer (ESIPT). We systematically investigate competing excited state decay channels in a total of eleven crystals to evaluate the factors needed for efficient ESIPT fluorophores, aided by a full evaluation of the crystal structures, exciton coupling, and exciton hopping rates. We show that in addition to the restriction of nonradiative pathways, an efficient ESIPT is essential to maximise the QE in the solid state. This extensive study of structure-property relationships for fluorophores based on the ESIPT mechanism bridges the understanding of molecular photophysics with crystal structure, accelerating the development of highly efficient solid state emitters.

File list (2)

paper_ChemRxiv.pdf (3.66 MiB)

view on ChemRxiv • download file

Supporting_Info.pdf (4.82 MiB)

view on ChemRxiv • download file 


\title{
Molecular and Crystalline Requirements for Solid State Fluorescence Exploiting Excited State Intramolecular Proton Transfer
}

\author{
Michael Dommett, Miguel Rivera, Matthew T. H. Smith, and Rachel \\ Crespo-Otero*
}

School of Biological and Chemical Sciences, Queen Mary University of London, London

E-mail: r.crespo-otero@qmul.ac.uk

Phone: +44 (0)20 78828404

\begin{abstract}
Aggregation induced emission offers a route to the development of emissive technologies based on solely organic systems. However, maximising fluorescence quantum efficiencies (QE) is a formidable challenge in attaining first-principles materials design, due to the interplay between the electronic structure of the chromophore and the molecular crystal. The identification of radiative and nonradiative channels, and how these are affected by aggregation, can rationalise the emissive properties of materials and aid in the design of yet more efficient fluorophores. In the current work, we examine the mechanism behind aggregation induced emission in two related families of compounds with lasing properties, which undergo excited state intramolecular proton transfer (ESIPT). We systematically investigate competing excited state decay channels in a total of eleven crystals to evaluate the factors needed for efficient ESIPT fluorophores, aided by a full evaluation of the crystal structures, exciton coupling,
\end{abstract}


and exciton hopping rates. We show that in addition to the restriction of nonradiative pathways, an efficient ESIPT is essential to maximise the QE in the solid state. This extensive study of structure-property relationships for fluorophores based on the ESIPT mechanism bridges the understanding of molecular photophysics with crystal structure, accelerating the development of highly efficient solid state emitters.

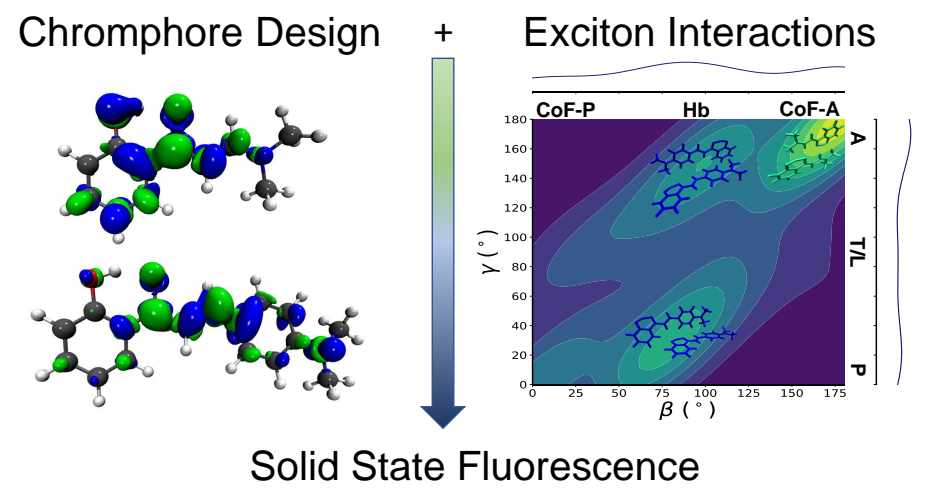

\section{Introduction}

Luminescent organic materials have technological promise for applications such as lightemitting diodes, field effect transistors, imaging and detection, and solid state lasers. ${ }^{1-7}$ Molecular organic systems offer the opportunity to tune absorption and emission characteristics for a desired application, through chromophore modification and crystal engineering. However, such control, at least from a design point of view, remains challenging due to the complex structure-property relationships in molecular crystals.

Optimising luminescence quantum efficiencies (QE) is imperative to making such devices and materials viable for use. Aggregation caused quenching (ACQ) has traditionally been the first obstacle to overcome in this process, since fluorescence quenching is highly common in conjugated aromatic systems as $\pi \pi$ interactions arise in the solid state. In past years researchers have developed a strategy to overcome ACQ via aggregation induced emission (AIE), where fluorescence is turned on upon molecular aggregation. ${ }^{8,9}$ This can require com-

plex and laborious substituent modification to inhibit nonradiative decay, in particular due 
to the difficulties in crystal structure design and the ensuing trial-and-error optimisation process.

Numerous mechanisms have been proposed to account for AIE, due to the variety of reported systems, including J-aggregate formation, suppression of Kasha's rule of fluorescence, excimer formation, restriction of intramolecular motions (RIM), and restricted access to conical intersections (RACI). ${ }^{7,10-16}$ Theoretical investigation into the AIE mechanism has been led by the groups of Peng and Shuai, who have developed formalism based on the Fermi Golden Rule approach to calculate radiative and nonradiative decay rates in AIE systems. ${ }^{17-23}$ The work of Blancafort et al. has shown that $\mathrm{S}_{1}-\mathrm{S}_{0}$ conical intersections (CIs) also play a role in the solid state. ${ }^{15,16}$ These theoretical frameworks have enabled researchers to understand the emitting properties of a myriad of systems. ${ }^{24}$

As well as the electronic properties of the chromophore, intermolecular interactions become photophysically important in the solid state due to the dense packing in organic crystals. Since the excited state is often localised on just a few molecular units, this effect is commonly examined through Kasha's two state exciton theory. ${ }^{7,10,25-27}$ Based on Kasha's model, dimers are generally assigned as H-aggregates based on the Coulomb interaction of transition dipole moments aligning side-by-side, whilst J-aggregates arise when a slip in the $x$-plane (long axis) occurs. At small intermolecular distances however, the sole use of the Coulomb interaction neglects the important contribution of short-range coupling effects, resulting in interferences which cannot be described by an electrostatic interpretation alone. ${ }^{26}$ Furthermore, when there is considerable perturbation between the Franck-Condon and the equilibrated excited state, Kasha's interpretation of H- and J-aggregates is less clear.

In this work we examine both the electronic properties of the chromophore, the intermolecular relationships in the molecular crystal, and the crystal structure, to rationalise the observed fluorescence behaviour for a set of related organic materials undergoing excited state intramolecular proton transfer (ESIPT). ESIPT involves a four level photocycle between enol (E) and keto $(\mathrm{K})$ tautomers triggered by electronic excitation in the $\mathrm{E}$ form. 
ESIPT followed by emission from the resultant $\mathrm{K}^{*}$ minimum (on the excited state PES) affords a large Stokes shift, separating the absorption and emission bands. Competition between $\mathrm{E}^{*}$ and $\mathrm{K}^{*}$ emission can be tuned, for example, through substituent modification and choice of solvent. ${ }^{28-33}$

Chalcone-based compounds, comprising an aromatic ketone and enone system, have found application in both technological and biological settings. ${ }^{33-48}$ The lasing properties of ESIPT emitters based on chalcone skeletons has aroused attention recently. In this work we investigate the properties of 2'-hydroxchalcone (HC) derivatives, and their mono-aryl anologues based on 2-hydroxyphenylpropenone (HP). The systems and properties are summarised in Table $1 .{ }^{49-51}$

Table 1: Molecular structures and QEs $(\Phi)$ in the solid state ${ }^{49-51}$
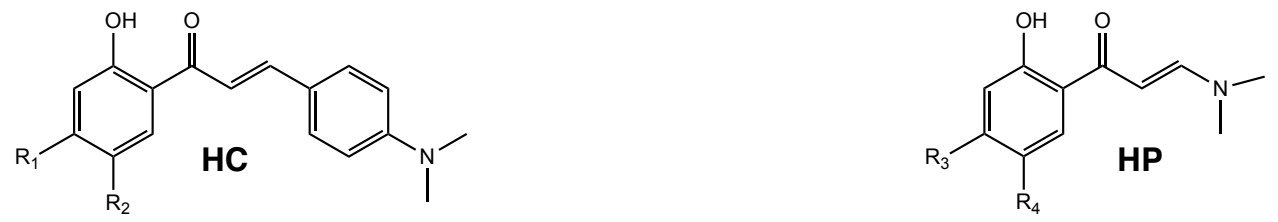

\begin{tabular}{llllllll}
\hline & $\mathrm{R}_{1}$ & $\mathrm{R}_{2}$ & $\Phi$ & & $\mathrm{R}_{3}$ & $\mathrm{R}_{4}$ & $\Phi$ \\
\hline HC1 & $\mathrm{H}$ & $\mathrm{H}$ & 0.32 & $\mathbf{H P 1}$ & $\mathrm{H}$ & $\mathrm{H}$ & 0.74 \\
HC2 & $\mathrm{CH}_{3}$ & $\mathrm{H}$ & 0.25 & $\mathbf{H P 2}$ & $\mathrm{F}$ & $\mathrm{H}$ & 0.84 \\
HC3 & $\mathrm{OCH}_{3}$ & $\mathrm{CH}_{3}$ & 0.26 & $\mathbf{H P 3}$ & $\mathrm{OCH}_{3}$ & $\mathrm{H}$ & 0.77 \\
HC4 & $\mathrm{H}$ & $\mathrm{CH}_{3}$ & $<0.01$ & $\mathbf{H P 4}$ & $\mathrm{H}$ & $\mathrm{F}$ & 0.72 \\
HC5 & $\mathrm{H}$ & $\mathrm{OCH}_{3}$ & $<0.01$ & & & & \\
HC6 & $\mathrm{F}$ & $\mathrm{H}$ & 0.41 & & & & \\
HC7 & $\mathrm{H}$ & $\mathrm{F}$ & 0.10 & & & & \\
\hline
\end{tabular}

The crystal packing, absorption and emission wavelength, and crucially the QE, are all dependent on the choice of substituent and number of aromatic rings. For structures based on HC, emission is dependent on the choice of substituent, which indirectly affects the packing mode. ${ }^{49}$ Previous theoretical investigations show that fluorescence is witnessed when the conical intersection is energetically inaccessible in the solid state. Furthermore, localisation of the excited state onto one molecule in the crystal promotes the crucial ESIPT step. ${ }^{52,53}$

Closely related to $\mathbf{H C}$ are the family of $\mathbf{H P}$ derivatives. ${ }^{50}$ In contrast to $\mathbf{H C}$, and other organic fluorophores, HP compounds contain only a single aryl group and have remarkable 
QEs, ranging from 0.72-0.84. This has been qualitatively attributed in experimental studies to the herringbone packing mode and molecular rigidity reducing nonradiative decay. Theoretical investigation can offer insight into the factors which confer this increase in QE, enabling further understanding into how to maximise fluorescence from first principles.

Herein we investigate the factors which mediate the increased fluorescence activity for HP compared to $\mathbf{H C}$ systems with particular focus on the role of the crystal structure and exciton couplings. Based on our previous work, we analyse the three step mechanism to enhance radiative decay in ESIPT solid state emitters; I) localisation of excited state to one monomer, II) bias for $\mathrm{K}^{*}$ decay over $\mathrm{E}^{*}$, III) an energetically inaccessible conical intersection. ${ }^{53}$ We use quantum chemical and hybrid simulations to address each of these steps to show how the HP systems have an increased potency in each one with respect to their $\mathbf{H C}$ counterparts, resulting in the $\mathbf{H P}$ systems' increased fluorescence in the solid state.

\section{Results and Discussion}

Excited state mechanisms in the solid state are controlled by intermolecular interactions, where the crystal structure determines the free volume available for the relaxation of the chromophore in the excited state. We begin by analysing the crystal structures of the eleven compounds. Each crystal can be examined initially from the perspective of a monomeric chromophore. To this end, we use Voronoi cell volumes $V_{\text {cell }}$ and van der Waals volumes $V_{v d W}$ to determine a Voronoi index $V_{i}=V_{\text {cell }} / V_{v d W}$, a metric indicating the normalised accessible volume for a monomer in the crystal. $V_{i}$ values (Figure S1) range from 1.111.53, showing that despite the substituent and packing differences, the each monomer in the system has between 10\%-50\% of its van der Waals volume to freely vibrate, rotate or translate. For the $\mathbf{H C}$ systems, the average $V_{i}$ is 1.48 , and 1.45 for the $\mathbf{H P}$ systems. The accessible volume for the $\mathbf{H C}$ systems shows greater variation but is overall slightly higher 
than for the less varied $\mathbf{H P}$ systems. The increased volume for the $\mathbf{H C}$ systems theoretically allow for greater nuclear relaxation in the excited state. However, reorganisation energies for the $\mathbf{H P}$ systems are larger than for $\mathbf{H C}$, showing the importance of the electronic effects over geometric considerations.

To determine the intermolecular relationships within the molcular crystals, we examine the topology of the molecular crystals of $\mathbf{H C}$ and $\mathbf{H P}$ families by considering dimer packing motifs. Crystal morphologies are commonly described qualitatively as herringbone, faceface, edge-tail, etc. Here we take a more of quantitative approach by constructing maps of each crystal, based on a geometric description of the dimers. These maps allow the topology of the crystal to be analysed graphically, as in Figure 2.
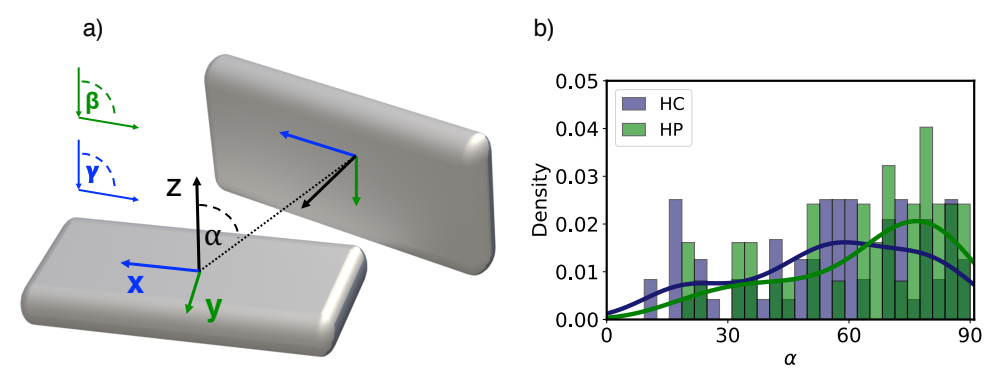

Figure 1: Panel a), left; schematic of two monomers $\mathrm{A}$ and $\mathrm{B}$, their centroids $C$, and the $\alpha, \beta$, and $\gamma$ angles used to classify dimer configurations. Panel b), right; distribution of $\alpha$ angles for dimers in $\mathbf{H C}$ and $\mathbf{H P}$ systems.

The dimer motifs are quantified through three angle variables, $\alpha, \beta$, and $\gamma$. These are depicted in Figure 1, and example motifs with associated angles are given in Figure S4. Three axes, $x, y, z$, are defined on each molecule $i$ and $j$ of the dimer, where $x$ and $y$ are the long and short axes of the molecule, and $z$ is the orthogonal vector. These vectors comprise an orthogonal basis to describe the dimer. The angles are then defined as:

- $\alpha$ : The azimuthal angle between the monomers shown as shown in Figure 1. Calculated as the angle between the $z$-axis located at the centroid of monomer $i$, and the vector connecting two centroids. $\alpha$ is calculated twice, once with each monomer as the reference. The smallest angle is chosen, provided $0^{\circ} \leq \alpha \leq 90^{\circ}$. 
- $\beta$ : The angle between the two short-axis vectors $y$ of each molecule, shown in green in Figure 1. $\beta$ ranges from $0^{\circ}$ to $180^{\circ}$, tracking whether monomers are aligned cofacially parallel $\left(\beta=0^{\circ}, \mathrm{CoF}-\mathrm{P}\right)$, or cofacially antiparallel $\left(\beta=180^{\circ}, \mathrm{CoF}-\mathrm{A}\right)$, or in a herringbone edge-face manner $\left(90^{\circ}, \mathrm{Hb}\right)$, and all configurations in between. $\beta$ is commonly described as the "herringbone" angle.

- $\gamma$ : The angle between the long-axis vectors $x$, ranging from $0^{\circ}$ (parallel, $\mathrm{P}$ ) to $180^{\circ}$ (antiparallel, A). At $y=90^{\circ}$, the dimer is T- or L-shaped, dependent on the $x$-slip.

In Figure $1 \mathrm{~b}$ the distribution density of the $\alpha$ angle is shown for the $\mathbf{H C}$ and $\mathbf{H P}$ systems. The distribution is heavily skewed towards $90^{\circ}$, indicating that for the majority of dimers, there is little overlap between the centroids of the monomers. As such, it can be expected that in each molecular crystal there are few dimers with the perfect overlap, with most undergoing a displacement in $x$ or $y$. This is common for aromatic systems. ${ }^{54}$

Figure 2 shows the dimer distribution densities for the $\beta$ and $\gamma$ angles for $\mathbf{H C}$ (top row) and HP (bottom row). Key regions are highlighted as an example in the upper plot of panel a); for example at $\beta=90, \gamma=0$, a herringbone $(\mathrm{Hb})$ stack is witnessed with the long axes arranged in parallel. For both $\mathbf{H C}$ and $\mathbf{H P}$ systems, the majority of dimers have $\beta$ and $\gamma$ angles close to $180^{\circ}$ (top right of plot), indicating that the most common dimer configuration is a cofacial arrangement where the carbonyl groups align antiparallel, at opposing ends of the molecule from eachother. This results in an antiparallel alignment of the $\mathrm{S}_{1}$ transition dipole moment of each monomer.

However, as panel b) shows, when the $\alpha$ angle is used as a filter, the configurations with acute azimuthal angles, and therefore closest in space, are mostly herringbone in nature for HC, with carbonyl groups at the same $\left(\gamma=0^{\circ}\right)$ or opposite ends of the dimer $\left(\gamma=180^{\circ}\right)$. In HC1-3 and HC6, the herringbone is the most common dimer, but does not occur at all in the HP systems, as shown in Table S2. For example, HC1 has three herringbone-like dimers and one cofacial dimer. With the largest unit cell and the conformational flexibility of the methoxy group, HC5 has the greatest variety of dimers, with nine herringbone, ten 
T-shape, and three cofacial types.

The cofacial arrangements favoured by the Kasha model occur at large slip displacements in the $x$ or $y$ plane $\left(\alpha>60^{\circ}\right)$, and are more like edge-edge coplanar arrangements rather than the well-known $\pi$-stack. Only in HC5 is there significant cofacial $\pi$-stacking between dimers, with other cofacial arrangements in $\mathbf{H C}$ and $\mathbf{H P}$ having larger $x$-slip. For $\mathbf{H C}$ compounds, $63 \%$ of the cofacially aligned dimers have a $x$-slip of less than half a molecule, whereas $68 \%$ of cofacially-algined HP dimers have a $x$-slip of more than half a molecule (Figure S2). In particular, the three cofacial dimers of HC5 have excellent alignment allowing for strong intermolecular interactions, with slip distances of less than half a molecule and all with $\alpha<40^{\circ}$.
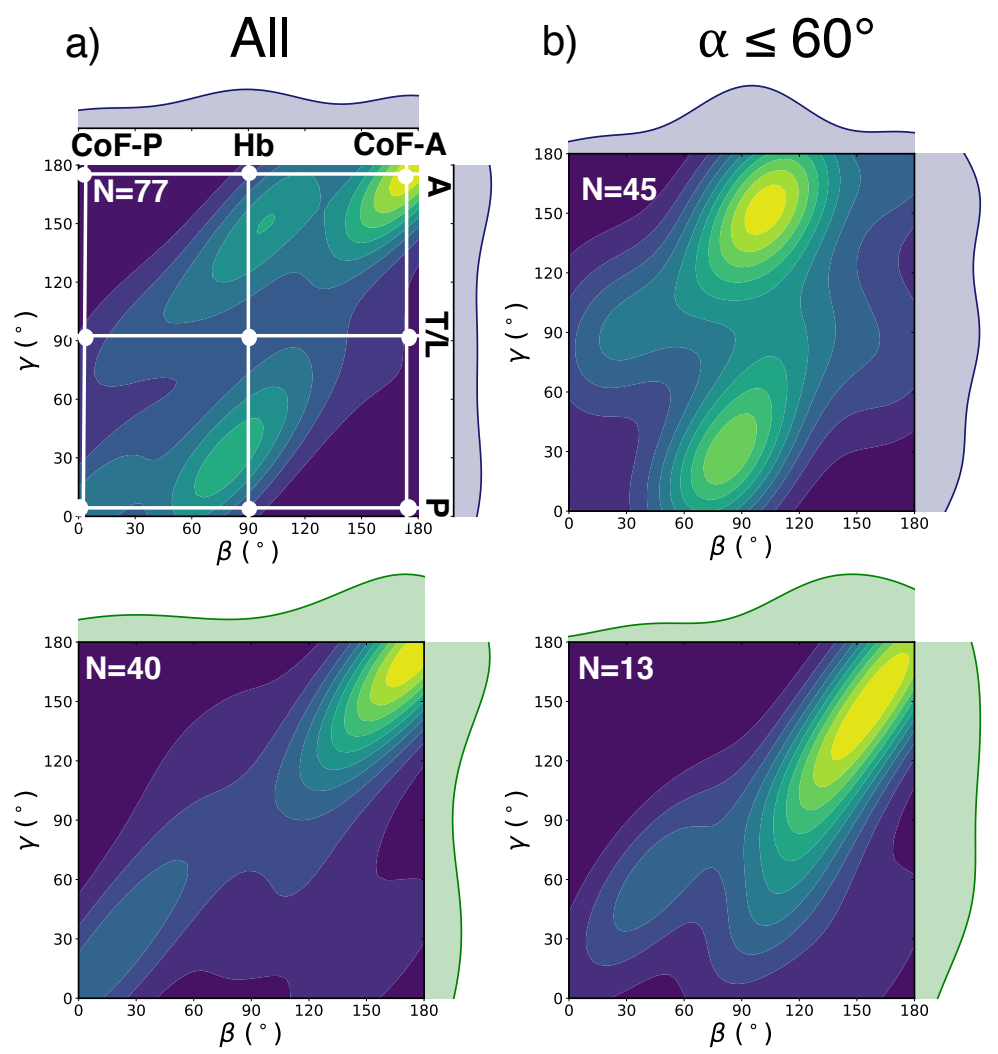

Figure 2: Panel a), left; Probability density map of the $\beta$ and $\gamma$ angles for $\mathbf{H C}$ (top) and HP (bottom) dimers. Key configurations are labelled on the axes, as explained in the text. Panel b), right; probability density for the subset of dimers where $\alpha \leq 60^{\circ}$.

In the $\mathbf{H P}$ systems with $\alpha \leq 60^{\circ}$, configurations lie on the diagonal (Figure 2)b, bottom), 
indicating dimers centred around a T-shape, which move to L-shaped when $\alpha \geq 60^{\circ}$. Overall, the significant dimer arrangement in $\mathbf{H P}$ compounds is a $\mathrm{T}$ shape, with the majority of cofacial arrangements having a large $x$ or $x$-slip with minimal $\pi \pi$ interactions due to the single aryl groups aligning at $y=180^{\circ}$. The $\alpha$ angle is generally larger than in $\mathbf{H C}$ with an average of $64^{\circ}$ compared to $55^{\circ}$, inhibiting short-range intermolecular interactions. However, in all HP systems, there is at least one prominent cofacial dimer which promotes exciton coupling, as we show in the next section.

\section{Intermolecular interactions in the molecular crystal}

Competing with localisation of the excited state necessary for ESIPT is exciton hopping. This competition can delay or even prevent proton transfer. ${ }^{53}$ Exciton transport can occur through coherent or incoherent hopping between molecular sites, and thus it is important to understand the possible intermolecular transport channels in the $\mathbf{H C}$ and $\mathbf{H P}$ systems. For the dimers discussed above, the exciton coupling $J_{i j}$ between monomers $i$ and $j$ is calculated with Troisi's diabatization scheme based on the orthogonal transformation of adiabatic states to diabatic states. ${ }^{55,56}$ This coupling scheme incorporates both short-range and Coulombic coupling mechanisms.

Considering the close packing of chromophores, we extended this method to asses the effect of a third monomer $k$ on the exciton coupling, where the coupling $J_{i j}$ between monomers $i$ and $j$ incorporates the effect of monomer $k$, which can quantified through comparison of the dimeric and trimeric coupling. However, we found that this made very little difference to the coupling in HC1, HC5 and HP1 compared to the kind of coupling modulations expected due to thermal fluctuations. ${ }^{55,57}$ This can be attributed to the excited state being delocalised over no more than two monomers of a trimer (Figures S9 and S10).

The exciton couplings for all dimers and the identity of the dimer with the largest $J$ are shown in Figure 3. Corroborating the robustness of the method, Figure S11a shows the coupling reproduces the supramolecular coupling of half of the energy splitting between $\mathrm{S}_{1}$ 
and $\mathrm{S}_{2}$ of the dimer. In $\mathbf{H C 1 - 4}$, where the closest packed dimers are herringbone in nature, similar dimer configurations are present. The largest coupling occurs in $\mathbf{H C 1}$, with $J=108$ meV. For HC-2, the dimer with the largest coupling is is an edge-edge stacked dimer with $\alpha=87^{\circ}$, where poor alignment reduces the coupling to $J=79 \mathrm{meV}$. HC3 and HC4 have similar herringbone stacking arrangements, where the largest couplings are $80 \mathrm{meV}$ and 93 meV respectively. In HC5-7, face-face stacked dimers are more prevalent in the crystal structure. The size of the coupling in HC6 is reduced compared to HC5 due to the $y$ displacement of one the monomers, with values of $148 \mathrm{meV}$ and $113 \mathrm{meV}$. A significant drop off to $83 \mathrm{meV}$ is seen for $\mathbf{H C 7}$ due to an increased $x$-slip in the cofacial dimer.

In the HP compounds, the large $\alpha$ and $x$-slip values, and the smaller molecular size, reduce the average coupling compared to $\mathbf{H C}$. In each $\mathbf{H P}$ derivative there exists one close packed, cofacial dimer which exhibits the largest coupling. For $\mathbf{H P 1}$, this is relatively small at just $48 \mathrm{meV}$ due to the $x$-slip. However, in HP2-3, the crystal structures afford more efficient cofacial stacking with $x$-slip values of only $1 \AA$, resulting in the largest couplings of all investigated systems, with $J=157 \mathrm{meV}$ for HP2 and $J=147 \mathrm{meV}$ for HP3.

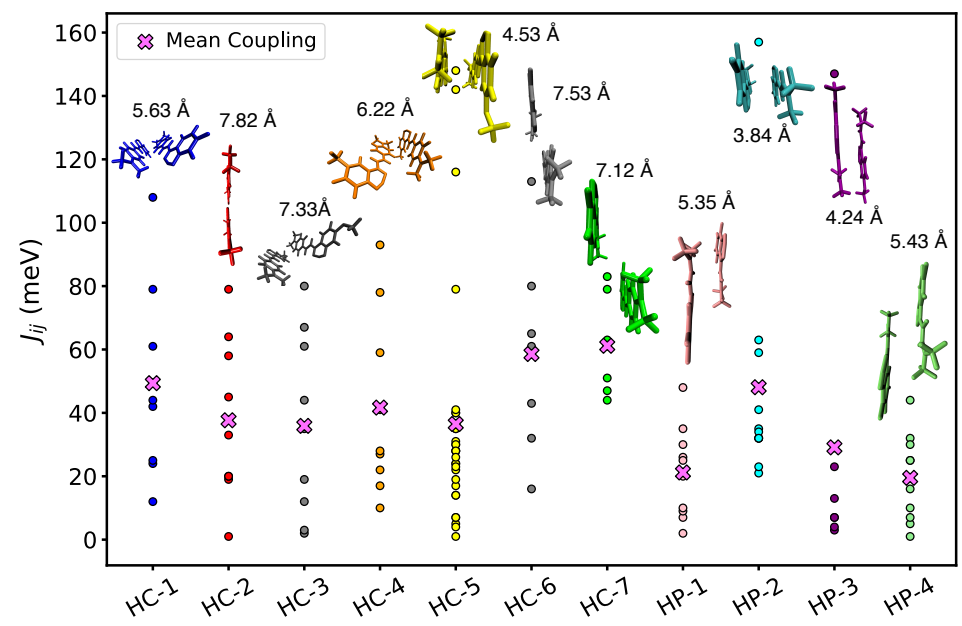

Figure 3: Exciton couplings $J$ between monomers $i$ and $j$ in the dimers identified in $\mathbf{H C}$ and HP. The mean coupling is also shown, along with the distance in angstroms between the constituent monomer centroids.

We assign the dimers as $\mathrm{H}$ - or J-aggregates based on the oscillator strength of the $\mathrm{S}_{1}$ and 
$\mathrm{S}_{2}$ excitation. In the Kasha model, for a perfectly stacked dimer with no $x$-slip, the oscillator strength of the $\mathrm{S}_{2}$ state should be double that of the monomer state. These systems generally fit the Kasha model, as when the $x$-slip is zero, a linear model (SI section S5) predicts an enhanced $\mathrm{S}_{2}$ intensity of 2.10 for the HCs and 1.83 for the HPs. With increasing $x$-slip, the difference in oscillator strength between the two states decreases until the inversion to J-aggregates is witnessed $\left(f_{S_{1}}>f_{S_{2}}\right)$. For the $\mathbf{H C s}$ this occurs at a $x$-slip of $52 \%$ of the monomer length and at $46 \%$ of the monomer length for the HCs.

For almost all systems, the number of $\mathrm{H}$ - and J-aggregates is very similar (Table S5). For HC-4, HC-5 and HC-7 the H-aggregate population grows to 78\%, $66 \%$ and $100 \%$, respectively, suggesting that an increase in $\mathrm{H}$-aggregates may be a cause for the smallest quantum yields in the solid state. However, this will be relevant only if there is a significant population of the $\mathrm{E}^{*}$ channel.

\section{Exciton localisation vs. hopping: the role of nuclear reorganisation and proton transfer}

Once the system is in the excited state, relaxation can happen through the $\mathrm{K}^{*}$ or $\mathrm{E}^{*}$ channels (or both). These mechanisms compete with exciton hopping, which prevents localisation of the electron density. For fluorescence to occur from the $\mathrm{K}^{*}$ state, the exciton must localise onto one monomer to enable ESIPT. ${ }^{53}$ Exciton hopping rates $\nu_{i j}$ between monomers $i$ and $j$ in a molecular crystal can be calculated based on a Marcus hopping scheme,

$$
\nu_{i j}=\frac{J_{i j}^{2}}{\hbar} \sqrt{\frac{\pi}{\lambda k_{B} T}} \exp \left[-\frac{\lambda}{4 k_{B} T}\right]
$$

where $k_{B}$ is the Boltzmann constant, $\hbar$ is the reduced Planck constant, $\mathrm{T}$ is the temperature $(298 \mathrm{~K})$, and $\lambda$ is the reorganisation energy. The activation energy can be approximated as 
$\lambda / 4 .{ }^{58,59}$ The reorganisation energy $\lambda$ of Equation 1 is calculated in the adiabatic regime via

$$
\begin{aligned}
\lambda_{A} & =\lambda_{e x}+\lambda_{g} \\
& =\left(E_{e x}^{*}-E_{e x}\right)+\left(E_{g}^{*}-E_{g}\right)
\end{aligned}
$$

where $E_{g}$ and $E_{e x}$ are ground state energies at the $\mathrm{S}_{0}$ and $\mathrm{S}_{1}$ minima, and $E_{g}^{*}$ and $E_{e x}^{*}$ are the corresponding excited state energies at respective minima. Herein, we consider the reorganisation energies involved in the relaxation to the $\mathrm{K}^{*}$ and $\mathrm{E}^{*}$ minima (Keto and Enol regimes respectively). $\lambda$ can also be calculated using normal mode analysis (see SI section S2). However, for HC1, HC5, and HP1, it is found that $\lambda_{N M}$ overestimates the reorganisation energy with respect to $\lambda_{A}$, so we use $\lambda_{A}$ in the following.

Solid state reorganisation energies $\lambda_{A}$ in keto and, when located, enol minima were calcu-

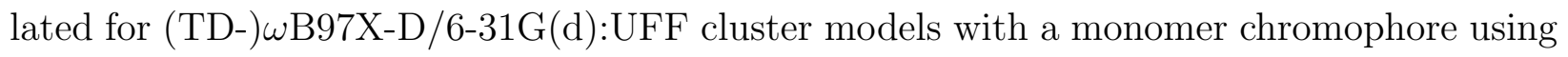
Equation 2. Figure 4 shows the coupling, reorganisation energy and the associated exciton hopping rate (using a log scale) for each dimer. The hopping rate $\nu$ is ultrafast in the enol regime $(\mathbf{H C 1}-\mathbf{4}, \mathbf{6 , 7})$, with the highest rates in the region between $1 \times 10^{13} \mathrm{~s}^{-1}$ and $6 \times 10^{13} \mathrm{~s}^{-1}$ due to the planar conformation conferring relatively low $\lambda_{A}(244 \mathrm{meV}$ on average). The highest and lowest $\nu$ for $\mathbf{H C} 1$ are $4.18 \times 10^{13} \mathrm{~s}^{-1}$ and $5.17 \times 10^{11} \mathrm{~s}^{-1}$, while the rate of ESIPT in the molecular crystal, through time resolved spectroscopy, is $3 \times 10^{11} \mathrm{~s}^{-1}$. 33 Intramolecular hopping is therefore expected to compete with ESIPT, which requires localisation of the excited state to one site in the molecular crystal. Due to similarity of the electronic and crystal structures, this should also be the case for $\mathbf{H C 2 - 3 , 6 , 7}$, hence opening nonradiative intermolecular decay channels for these systems and a source of quantum yield leak. For HC4 and HC5, there is a significant bias towards proton transfer because of a larger reorganisation energy ( $935 \mathrm{meV}$ and $978 \mathrm{meV}$ respectively).

In vacuum, an $\mathrm{E}^{*}$ minimum is found on the PES for $\mathbf{H P}$ systems, where the relaxation mode is via partial trans-cis isomerisation about the aliphatic double bond. This minimum was optimised for HP1 in the solid state and it was found to have a reorganisation energy 
of $2.19 \mathrm{eV}$ compared to $1.31 \mathrm{eV}$ for the $\mathrm{K}^{*}$ state. As a direct consequence, the hopping rate for such a large $\lambda$ is $1.44 \times 10^{4} \mathrm{~s}^{-1}$. For comparison, the smallest hopping rate for $\mathbf{H C 1}$ is $5.17 \times 10^{11} \mathrm{~s}^{-1}$. Exciton hopping is therefore reduced in $\mathbf{H P}$ due to the relatively small exciton coupling and the large reorganisation energy in the enol state, with the bias for ESIPT on account of the electron density redistribution rendering ESIPT the most favourable relaxation channel in the solid state.

In the $\mathrm{K}^{*}$ channel, hopping rates in $\mathbf{H P}$ are systematically lower than in the $\mathbf{H C}$ family. On average, $\nu=7.51 \times 10^{8} \mathrm{~s}^{-1}$ in the HPs compared to $7.61 \times 10^{10} \mathrm{~s}^{-1}$ The highest rate in the HP family is $1.33 \times 10^{10} \mathrm{~s}^{-1}$, compared to a $1.64 \times 10^{12} \mathrm{~s}^{-1}$ in the HCs. This is on account of the high stability of the $\mathrm{K}^{*}$ minimum in the HPs producing a large reorganisation energy.

The ESIPT bias in HP derivatives can be understood through analysis of electronic transition densities, where a loss of electron density at the hydroxyl oxygen increases the acidity of the proton with respect to the $\mathbf{H C}$ systems, where this loss is not present, except in HC5 (Figure S6). NBO charge analysis of the phenol oxygen shows that $\Delta \mathrm{q}$ increases from +0.01 to +0.05 to +0.09 for HC1, HC5 and HP1 respectively. This increases the bias for ESIPT in HC5 and HP1 due to the increased acidity of the transferring proton, as shown by the excited state PES relaxed geometry scan in Figure S9.

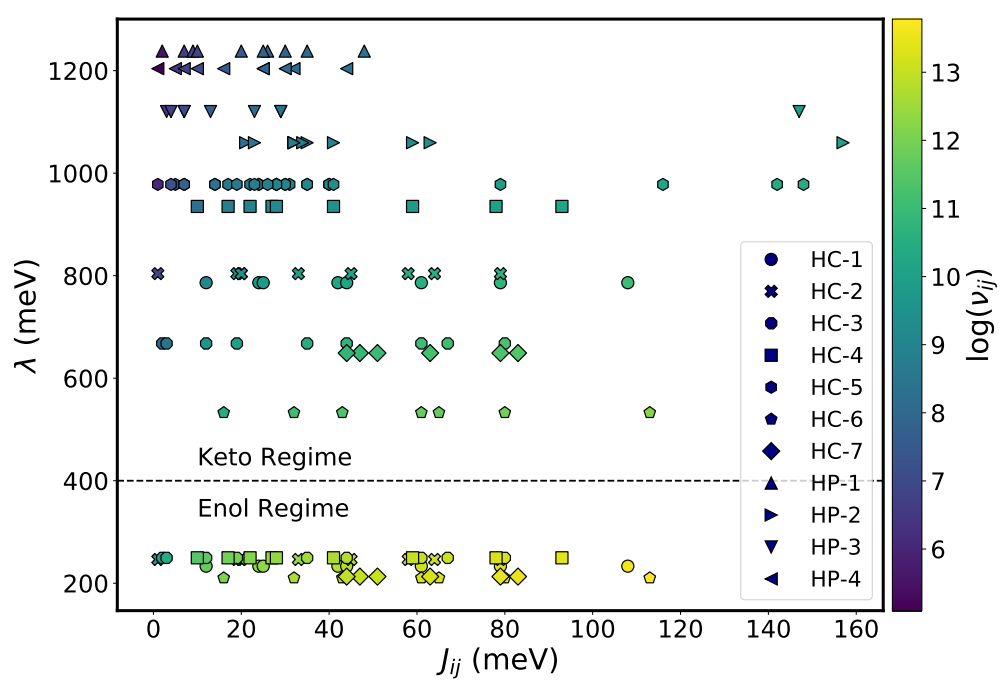

Figure 4: Colourmap of the exciton hopping rate $\nu_{i j}$ on a $\log _{10}$ scale, as a function of the exciton coupling $J_{i j}$ and the reorganisation energy $\lambda$, calculated via Equation 1. 


\section{The AIE Mechanism}

We compute the radiative decay rates $k_{r}$ from the $\mathrm{E}^{*}$ and $\mathrm{K}^{*}$ states in ONIOM (TDDFT)

by computing the fluorescence spectrum with Newton-X and calculating $k_{r}$ through ${ }^{60,61}$

$$
k_{r}=\frac{1}{\hbar} \int \Gamma_{r}(E) d E
$$

where $\Gamma_{r}$ is the rate of spontaneous emission per molecule per unit of angular frequency between $E / \hbar$ and $(E+d E) / \hbar .^{62}$ The spectra for HC1, HC5 and HP1, which are the extreme cases, are given in Figure S15 and radiative rates for HC1, HC5 and HP1 are given in Table 2 .

For HC1, $k_{r, K *}$ is comparable with $\mathbf{H P 1}$ in both $\mathrm{E}^{*}$ and $\mathrm{K}^{*}$. However, HC1 exhibits lower quantum efficiency, owing to the competing exciton hopping in the enol state, which will inhibit localisation and thus ESIPT. Thus in HC1 there is competition between delocalisation, emission from $\mathrm{E}^{*}$, and emission from $\mathrm{K}^{*}$. Due to the proximity of the $\mathrm{E}^{*}$ band to the absorption at $3.3 \mathrm{eV}$, only $\mathrm{K}^{*}$ fluorescence will significantly contribute to the quantum yield.

The RIM interpretation of AIE prescribes that switch-on of fluorescence upon aggregation is due to dampening of rotational modes, which dissipate the excited state nonradiatively, as witnessed by a reduction in the HR factors. While HC1, HC5 and HP1 show this effect, HC1 and HP1 show AIE while HC5 is dark in both dispersed and aggregated forms. The dampening of the HR factors does not result in luminescence in HC5, suggesting that the excited state wavepacket decays through the MECI. ${ }^{53}$ Furthermore, due to the anharmonicity of the PES, the validity of the scheme in these cases is not clear and $\lambda_{A}$ is used in the main text.

For $\mathbf{H P 1}$, emission from $\mathrm{E}^{*}$ is negligible and emission is expected from the $\mathrm{K}^{*}$ state. The highly distorted $\mathrm{E}^{*}$ minimum, as discussed in the previous section, is expected to play little role in the photochemistry and population transfer to $\mathrm{K}^{*}$ will dominate. The radiative 
rate from $\mathrm{K}^{*}$ is of similar magnitude to $\mathbf{H C 1}$, and as such the higher quantum yield of $\mathbf{H P 1}$ is on account of the lower nonradiatve decay rate and lack of other competitive pathways, such as exciton hopping.

Table 2: Radiative decay rates $k_{r}$ in the solid state in the enol $\left(\mathrm{E}^{*}\right)$ and keto $\left(\mathrm{k}^{*}\right)$ regimes for $\mathbf{H C 1}$, HC5, HP1. All rates in $\mathrm{s}^{-1}$.

\begin{tabular}{lll}
\hline System & $k_{r, E *}$ & $k_{r, K *}$ \\
\hline HC1 & $4.68 \times 10^{8}$ & $2.99 \times 10^{8}$ \\
HC5 & - & $9.54 \times 10^{7}$ \\
HP1 & $5.58 \times 10^{6}$ & $1.10 \times 10^{8}$ \\
\hline
\end{tabular}

For the HP family the stabilisation arising from ESIPT is larger than for the $\mathbf{H C}$ systems. For the HC systems, reorganisation energies due to ESIPT range from $532 \mathrm{meV}$ (HC3) to 978 meV (HC5). However, by modifying the chromophore molecular structure through removal of the second aryl group, the bias towards ESIPT is increased for the $\mathbf{H P}$ systems due to the instability of the planar $\mathrm{E}^{*}$ conformer and the high stablity of the $\mathrm{K}^{*}$ conformer. No stable planar $\mathrm{E}^{*}$ minimum can be located in the crystal such is the bias for ESIPT, with reorganisation energies ranging from $1.06 \mathrm{eV}$ (HP2) to $1.23 \mathrm{eV}$ (HP1). As such, in the keto state, the hopping rate is up to three orders of magnitude lower than the for the HC systems in the enol channel. Due to the increased organisation energy arising from tautomerism, localisation and ESIPT will be favoured over exciton hopping and a larger Stokes-shifted emission is witnessed for the HP family.

To achieve an efficient emissive response in the solid state, in addition to an efficient population of the $\mathrm{K}^{*}$ tautomer, nonradiative decay channels should be blocked. HC1, HC5 and HP1 show a reduction of Huang-Rhys factors and reorganisation energies associated with low-frequency motions in the solid state in contrast with the isolated chromophores (see section $\mathrm{S} 2$ in the $\mathrm{SI}$ ). Consequently, the RIR model does not explain their different photochemical behaviour and we need to consider the accesibility of the conical intersections (RACI model).

It has been shown that for HC1, fluorescence is possible due to the high energy conical 
intersection in the solid state. On the other hand, for HC5 and HC4, whilst ESIPT is more efficient than in $\mathbf{H C 1}$, the QE is very small and AIE is not seen. ${ }^{49}$ This can be attributed to dominance of nonradiative decay as a result of a low-lying MECI being classically accessible post electronic excitation. ${ }^{53}$ While intermolecular factors play a role in the conformation of the MECI, the energetic accessibility is determined by the electronic structure of the chromophore. To asses the accessibility of the MECI in the solid-state in HP1, we construct the excitation-decay pathway in the using QM:MM cluster models. The calculated PES for HP1 in vacuum and the solid state is shown in Figure 5.

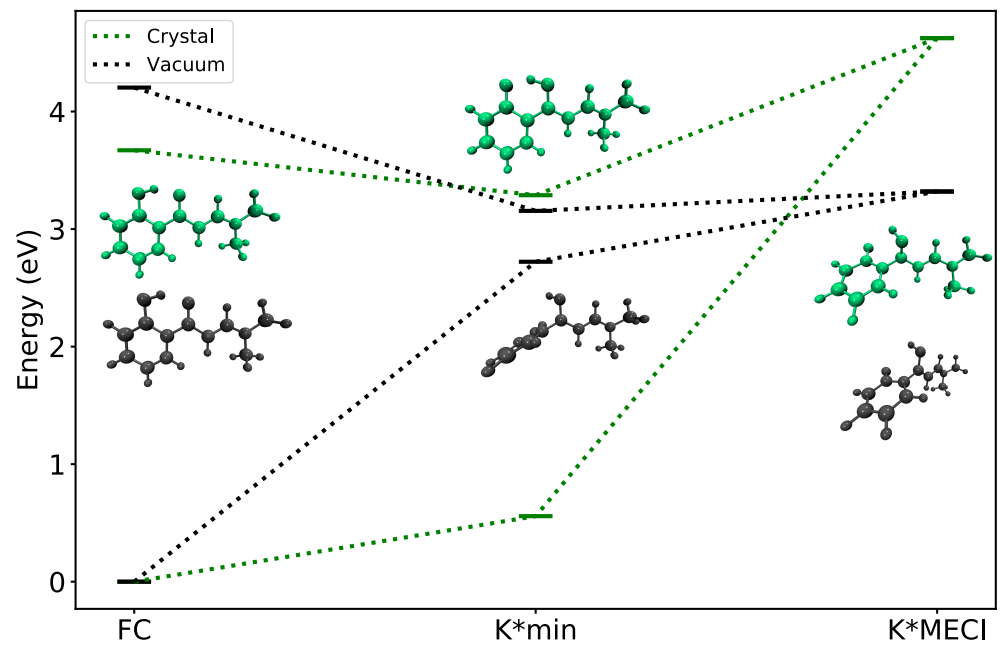

Figure 5: Calculated energies and geometries at critical points on PES. Crystal geometries obtained with ONIOM((TD)- $\omega$ B97X-D/6-31G(d):AMBER), with energies calculated at MS-3-CASPT2(12,11):AMBER. Vacuum geometries obtained with (TD)- $\omega$ B97X-D/6$311++\mathrm{G}(\mathrm{d}, \mathrm{p})$ and energies obtained with MS-3-CASPT2(12,11). The average energy of the $\mathrm{S}_{1}$ and $\mathrm{S}_{0}$ states is shown for the MECI.

At the MS-3-CASPT2(12,11)/6-31G(d):AMBER level, absorption for HP1 is calculated at $3.67 \mathrm{eV}(f=0.868)$, in fair agreement but blue-shifted by $0.41 \mathrm{eV}$ compared with the crystalline absorption maximum of $3.26 \mathrm{eV} .{ }^{50}$ Post photo-excitation, relaxation in $\mathrm{S}_{1}$ via ESIPT is expected to be dominant relaxation channel in HP1 due to the negligible oscillator strength $(f=0.016)$ of the $\mathrm{S}_{2}$ state, which is $n \pi^{*}$ in character. Fluorescence in the molecular crystal is centred at $2.34 \mathrm{eV}$, thus displaying a Stokes shift of $0.94 \mathrm{eV}$. The emission wavelength predicted at CASPT2 is $2.73 \mathrm{eV}$, again in fair agreement and with similar 
blue-shift as calculated for absorption.

The MECI in HP1 lies $1.46 \mathrm{eV}$ above the $\mathrm{K}^{*}$ minimum and $1.08 \mathrm{eV}$ above the bright absorption state. As such, it is classically inaccessible and HP emission can be attributed to the trapping of the excited state at the $\mathrm{K}^{*}$ minimum, followed by radiative decay. The degeneracy of the $\mathrm{S}_{1}$ and $\mathrm{S}_{0}$ states on going from CASSCF to CASPT2 is lifted, with a gap of $0.2 \mathrm{eV}$ at CASPT2 level. As the substituent effects in the crystalline samples are minor in the HP samples, it can be assumed that this mechanism can be applied to all four systems in the family. It would be of interest to synthesise a HP system to assess its AIE behaviour with a methoxy group the para position, as in HC5.

In Figure 6, the MECI geometries of $\mathbf{H C 1}, \mathbf{H C 5}$, and $\mathbf{H P 1}$ are compared. All three involve the pyramidalisation of the protonated carbonyl group combined with torsional rotation of the deprotonated phenol moeity. In $\mathbf{H C 1}$ and $\mathbf{H P 1}$, the compounds which undergo AIE due to the high energy of the MECI, the torsional angles are $49.6^{\circ}$ and $53.3^{\circ}$. In HC5, where the MECI is energetically accessible, the pyramidal distortion is only $26.7^{\circ}$. The same effect is seen in vacuum, where the MECI of HC5 is also onset at lesser distortion than the other systems, as discussed above and shown in Figure S13. Therefore the high energy MECIs of HC1 and HP1, are inherent to the electronic effects of the chromophore. Rather, the role of the packing and the crystalline environment as a whole is to promote efficient localisation of the excited state at the expense of exciton hopping the enol state. A largely increased QE is witnessed as a result of the high propensity for ESIPT in HP and the high energy MECI.

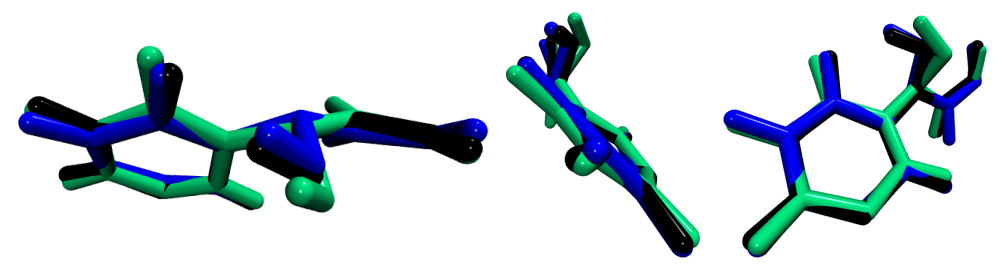

Figure 6: Overlaid structures of the MECI for HC1 (blue) and HC5 (black), and HP1 (green), shown from three viewpoints. Only the atoms shared by all three compounds are shown. 


\section{Conclusions}

In this work we have systematically evaluated the photo behaviour of a range of solid-state emitters based on the ESIPT mechanism. In the HC family of compounds, AIE is witnessed for five of the seven compounds, with QEs ranging from 0.1 to 0.41 . In the $\mathbf{H P}$ systems, which differ by containing only one aryl ring, all reported compounds are emissive in the solid state with QEs of 0.72-0.84. In each crystal structure, there exist a range of dimer motifs each with their own excitonic profile. In the $\mathbf{H C}$ systems, with the exception of HC5, the herringbone stacking is prominent, with exciton coupling enhanced by the two aryl rings promoting $\pi \pi$ interactions which are mostly absent in their HP counterparts,

In the $\mathbf{H C}$ compounds (except $\mathbf{H C 5}$, after photoexcitation to the $\mathrm{S}_{1}$ state, exciton hopping in the enol tautomer will compete with ESIPT on account of minimal electron density loss on the phenol oxygen and the stability of the planar enol tautomer, which results in only a small reorganisation energy. Here the the hopping rate is several orders of magnitude larger than in the $\mathrm{K}^{*}$ state and will allow nonradiative dissipation of the excited state. Conversely in the HP compounds, and HC5, the electron density loss is increased on the oxygen and ESIPT is more favourable, coupled with the planar $\mathrm{E}^{*}$ tautomer being unstable. The stability of the $\mathrm{K}^{*}$ state increases the reorganisation energy $\lambda$ of the chromophore and subsequently will increase the population of the ESIPT channel in these systems. The $\mathrm{K}^{*}$ state will be highly localised with minimal hopping. In HC1, emission from $\mathrm{E}^{*}$ will increase unfavourable self-absorption and contribute to the lower quantum yield compared to HP1.

The $\mathrm{K}^{*}$ minimum takes a planar conformation in the solid state, with considerable oscillator strength for emission back to $\mathrm{S}_{0}$. HP1 has an energetically inaccessible MECI, like HC1, whereas in HC5 the MECI is energetically accessible. As for HC1, the MECI for HP1 in the crystal takes a distorted, pyramidalised geometry and is energetically inaccessible in the decay path. As such, emission will occur from the planar $\mathrm{K}^{*}$ minimum for the $\mathbf{H P}$ compounds and with larger $\mathrm{QE}$, due to the increase in the population of the $\mathrm{K}^{*}$ channel. Such is the similarity in absorption and emission spectra in the HP family, this mechanism 
is expected to be independent of the substituents present in this study.

At this point, we can say that incorporation of the following features into the next generation of ESIPT emitters can move the field forward, based on the remarkable properties of the HP derivatives:

1. To maximise the population of the ESIPT channel, chromophore design should encourage a highly labile proton, where electronic excitation destabilises the $\mathrm{E}^{*}$ state.

2. Packing modes should limit $\pi-\pi$ interactions, which enable exciton coupling and delocalisation. However, if the ESIPT is favourable enough, the localisation inherent to the $\mathrm{K}^{*}$ state can overcome unfavourable stacking arrangements.

3. In the $\mathrm{K}^{*}$ form, solid state conical intersections are accessed via a combination of pyramidalisation and rotation. This can be made more unfavourable by tethering and chromophore design, where we have shown that simple measures such as scanning coordinates in vacuum can predict $\mathrm{K}^{*}$ stability.

This work connects the electronic, molecular picture with the crystalline regime for organic, light-emitting materials. In these ESIPT emitters, the intermolecular interactions dominate in the Franck-Condon regime at photoabsorption. Subsequently the electronic structure and the character of the excited state, inherently molecular properties, become dominant. The deconstruction of intra- and intermolecular factors here, connecting the chromophore with its crystal structure, offers a step forward in first principles design of solid state luminescent materials.

\section{Computational Methods}

Crystal structures of compounds HC1-7 and HP1-4 were obtained from the CCDC as described in references 49-51. Monomers of HC1-5 and HP1-4 were optimised in vacuum in 
the $\mathrm{S}_{0}$ and $\mathrm{S}_{1}$ enol and keto states at the (TD-) $\omega$ B97X-D/6-311++G(d,p) level of theory. Relaxed geometry scans of the torsional rotation angle in the keto $\mathrm{S}_{1}$ state $\left(\mathrm{K}^{*}\right)$ were performed for the same compounds. Proton migration scans of the ESIPT process were also performed in vacuum for $\mathbf{H C 1}$ and $\mathbf{H P 1}$. All scans were calculated at TD- $\omega$ B97X-D/6-31G(d).

Crystal structures of all HC and HP compounds were optimised using Quantum Espresso in the periodic DFT framework. ${ }^{63}$ Optimisation of each unit cell was carried out with DFTD2 (PBE) with a plane-wave cutoff of 30 Ry and ensuring Monkhorst-Pack k-point convergence in each case.

Exciton couplings $J_{i j}$ were calculated for dimers in each optimised crystal structure. A $2 \times 2 \times 2$ supercell was constructed for each system, starting from the optimised unit cell, and all unique dimers where identified and extracted. A dimer was defined as any molecular pair with an interatomic distance less than or equal to the van der Waals radii of the atoms plus a distance of $1.5 \AA$. This selection criterion has previously been used in similar applications. ${ }^{64}$ The coupling $J_{i j}$ between monomers $i$ and $j$ in unique dimers are calculated in Troisi's diabatization scheme based on the orthogonal transformation of adiabatic states to diabatic states based on the transition dipole moments. ${ }^{55,56}$

Crystalline emission spectra for HC1, HC5 and HP1 in $\mathrm{E}^{*}$ and $\mathrm{K}^{*}$ minima were simulated using the nuclear ensemble method as implemented in the NEWTON-X software suite. ${ }^{60} 100$ initial conditions were sampled from the harmonic frequencies calculated at

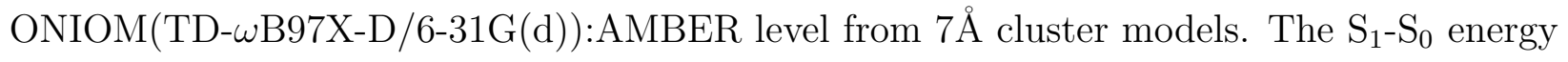
gap was computed for each initial condition in embedded point charges to reflect the positions of the MM charges. No MM-level energies were computed, and as such the fluorescence spectra are of only the electronic energies.

To calculate solid state reorganisation energies in the adiabatic approximation $\left(\lambda_{A}\right)$, for each compound we generated cluster models based on the $2 \times 2 \times 2$ supercell, where all molecules which lay within $20 \AA$ of the central chromophore were included in the cluster. Geometries were optimised in $\mathrm{S}_{1}$ and $\mathrm{S}_{0}$ states within the ONIOM protocol at $\omega \mathrm{B} 97 \mathrm{X}-\mathrm{D} / 6$ - 
31G(d):UFF using electronic embedding. MM charges were derived automatically using the QEq method. ${ }^{65}$ The UFF forecefield was chosen here due to the automatic charge assignment, allowing the highthroughput generation of structures and input files. In the cases of HC1-4 and HC6-7, $\lambda_{A}$ was calculated for both enol and keto pathways. For HC5 and HP1-4, only the $\lambda_{A}$ associated with keto relaxation was used since no $\mathrm{E}^{*}$ minimum was located in the monomer QM:MM relaxation. Reorganisation energies in the normal-mode approximation $\left(\lambda_{N M}\right)$ were calculated using the DUSHIN program for HC1, HC5 and HP1. ${ }^{66}$ Frequencies for an ONIOM-optimised monomer chromophore were calculated in an array of point charges representing the molecular crystal, at TD- $\omega$ B97X-D/6-31G(d) level. This lead to one imaginary frequency in each case.

The radiative rates $k_{r}$ in the molecular crystals of $\mathbf{H C 1}$, HC5 and HP1 were calculated by simulating the fluorescence spectrum using the nuclear ensemble method. Excited state frequencies at the $\mathrm{E}^{*}$ (where located) and $\mathrm{K}^{*}$ minimum for a monomer chromophore were calculated at ONIOM(TD- $\omega$ B97X-D/6-31G(d):AMBER) level of theory and used to generate 100 initial conditions using a Wigner distribution. For each initial condition the $\mathrm{S}_{1}-\mathrm{S}_{0}$ energy gap was calculated in a bed of point charges representing the MM atoms at TD- $\omega$ B97X-D/6$31 \mathrm{G}(\mathrm{d})$ level. Integration of the spectrum gives $k_{r}$ as described in reference 61 .

For HP1, the full excited state decay mechanism was established through QM:MM cluster models using density functional and multireference methods. A monomer and trimer chromophore at the centroid of the $20 \AA$ cluster were optimised in the ground and excited states at (TD-) $\omega$ B97X-D/6-31G(d):AMBER level of theory. The $\mathrm{S}_{1} / \mathrm{S}_{0} \mathrm{MECI}$ in both monomer and trimer cluster models were calculated using a modified version of the CIOpt algorithm. ${ }^{67,68}$

The MECI in the monomer cluster models was also obtained with the state-averaged complete active space self-consistent field method, employing the $S_{0}$ and $S_{1}$ states in the averaging. The active space consisted of 12 electrons in 11 orbitals (SA-2-CASSCF $(12,11)$ ), shown in Figure S11. The 6-31G(d) basis set was using for the QM region and the AMBER forcefield was used to describe the MM region. Calculating the MECI with CASSCF:MM ensures the 
validity of the TD-DFT:MM-calculated MECI. The potential energy profile was refined with multistate complete active space second-order perturbation theory (MS-3-CASPT2(12,11)/6$31 \mathrm{G}(\mathrm{d})$ :AMBER), incorporating the $\mathrm{S}_{0}, \mathrm{~S}_{1}$, and $\mathrm{S}_{2}$ states. The TD-DFT:MM geometries from the trimer models at the Franck-Condon, $\mathrm{S}_{1}$ minimum, and MECI were taken as the reference geometries, where the central molecule was taken for the CASPT2 calculation and the remaining two molecules of the trimer were added to the MM region. A three state average was used due to include the $\mathrm{S}_{2}$ electronic state at the Franck-Condon geometry. The orbitals chosen for the active space are shown in Figure S11. All density functional

calculations were performed in the Gaussian 09 suite of programs. ${ }^{69}$ CASSCF and CASPT2 calculations used OpenMolcas with the Tinker v.6.3.3 interface. ${ }^{70}$

To analyse the spatial environment for the monomers at the centre of the cluster models in the each crystal, Voronoi cells partition the crystal into molecular regions. These cells define all the points in space which are closer to the reference molecule than an exterior molecule. Dividing the Voronoi cell volume by the van der Waals volume gives a moleculeindependent Voronoi index $V_{i}$ for each crystal structure. To generate the Voronoi cells, a cluster of molecules was extracted from its crystalline positions. A real space grid was generated at an arbitrary resolution and at each point of this grid, the distance to each atom was calculated and scaled by the corresponding van der Waals radius. All voxels with the lowest scaled distance belonging to an atom of the central molecule were marked as belonging to the accessible of the molecule, resulting in an irregular polyhedron of finite volume. The analysis of volumes and dimer motifs is implemented in the package fromage. ${ }^{71}$

\section{Acknowledgement}

This work was supported by the EPSRC (EP/R029385/1). This research utilized Queen Mary's Apocrita HPC facility, supported by QMUL Research-IT, ARCHER UK National Supercomputing Service (EP/L000202) via the Materials Chemistry Consortium and the 
Thomas cluster (EP/P020194/1) via our membership to the Thomas Young Centre. The authors thank Jeff Reimers for supplying the DUSHIN code. The authors acknowledge the support from the School of Biological and Chemical Sciences at Queen Mary University of London.

\section{Supporting Information Available}

\section{References}

(1) Shih, P. I.; Chuang, C. Y.; Chien, C. H.; Diau, E. W. G.; Shu, C. F. Highly Efficient NonDoped Blue-Light-Emitting Diodes Based on an Anthrancene Derivative End-Capped with Tetraphenylethylene Groups. Adv. Funct. Mater. 2007, 17, 3141-3146.

(2) Huang, J.; Sun, N.; Dong, Y.; Tang, R.; Lu, P.; Cai, P.; Li, Q.; Ma, D.; Qin, J.; Li, Z. Similar or Totally Different: The Control of Conjugation Degree Through Minor Structural Modifications, and Deep-Blue Aggregation-Induced Emission Luminogens for Non-Doped OLEDs. Adv. Funct. Mater. 2013, 23, 2329-2337.

(3) Zhao, Z.; Li, Z.; Lam, J. W. Y.; Maldonado, J.-L.; Ramos-Ortiz, G.; Liu, Y.; Yuan, W.; Xu, J.; Miao, Q.; Tang, B. Z. High Hole Mobility of 1,2-bis[4-(diphenylamino)biphenyl4-yl]-1,2-diphenylethene in Field Effect Transistor. Chem. Commun. 2011, 47, 6924.

(4) Aldred, M. P.; Zhang, G.-F.; Li, C.; Chen, G.; Chen, T.; Zhu, M.-Q. Optical Properties and Red to Near Infrared Piezo-Responsive Fluorescence of a TetraphenylethenePerylenebisimideTetraphenylethene Triad. J. Mater. Chem. C 2013, 1,6709 .

(5) Li, Y.; Xu, L.; Su, B. Aggregation Induced Emission for the Recognition of Latent Fingerprints. Chem. Commun. 2012, 48, 4109-4111. 
(6) Kwok, R. T. K.; Leung, C. W. T.; Lam, W. Y. Biosensing by Luminogens with Aggregation- Induced Emission Characteristics. Chem. Soc. Rev 2015, 44, 4228-4238.

(7) Gierschner, J.; Park, S. Y. Luminescent Distyrylbenzenes: Tailoring Molecular Structure and Crystalline Morphology. J. Mater. Chem. C 2013, 1, 5818.

(8) Mei, J.; Leung, N. L. C.; Kwok, R. T. K.; Lam, J. W. Y.; Tang, B. Z. AggregationInduced Emission: Together We Shine, United We Soar! Chem. Rev. 2015, 115, 1171811940.

(9) Mei, J.; Hong, Y.; Lam, J. W. Y.; Qin, A.; Tang, Y.; Tang, B. Z. Aggregation-Induced Emission: The Whole is More brilliant Than the Parts. Adv. Mater. 2014, 26, 54295479 .

(10) Gierschner, J.; Lüer, L.; Milián-Medina, B.; Oelkrug, D.; Egelhaaf, H. J. Highly Emissive H-Aggregates or Aggregation-Induced Emission Quenching? The Photophysics ofAll-Trans Para-Distyrylbenzene. Journal of Physical Chemistry Letters 2013, 4, 2686-2697.

(11) Qian, H.; Cousins, M. E.; Horak, E. H.; Wakefield, A.; Liptak, M. D.; Aprahamian, I. Suppression of Kasha's rule as a mechanism for fluorescent molecular rotors and aggregation-induced emission. Nature Chemistry 2017, 9, 83-87.

(12) An, B. K.; Kwon, S. K.; Jung, S. D.; Park, S. Y. Enhanced Emission and its Switching in Fluorescent Organic Nanoparticles. Journal of the American Chemical Society 2002, 124, 14410-14415.

(13) Prlj, A.; Došlić, N.; Corminboeuf, C. How Does Tetraphenyl-Ethylene Relax from Its Excited States? Phys. Chem. Chem. Phys. 2016, 18, 11606-11609.

(14) Gao, Y. J.; Chang, X. P.; Liu, X. Y.; Li, Q. S.; Cui, G.; Thiel, W. Excited-state Decay Paths in Tetraphenylethene Derivatives. J. Phys. Chem. A 2017, 121, 2572-2579. 
(15) Peng, X.-L.; Ruiz-Barragan, S.; Li, Z.-S.; Li, Q.-S.; Blancafort, L. Restricted Access to a Conical Intersection to Explain Aggregation Induced Emission in Dimethyl Tetraphenylsilole. J. Mater. Chem. C 2016, 4, 2802-2810.

(16) Li, Q.; Blancafort, L. A Conical Intersection Model to Explain Aggregation Induced Emission in Diphenyl Dibenzofulvene. Chem. Commun. 2013, 49, 5966-5968.

(17) Li, W.; Zhu, L.; Shi, Q.; Ren, J.; Peng, Q.; Shuai, Z. Excitonic coupling effect on the nonradiative decay rate in molecular aggregates: Formalism and application. Chem. Phys. Lett. 2017, 683, 507-514.

(18) Zheng, X.; Peng, Q.; Zhu, L.; Xie, Y.; Huang, X.; Shuai, Z. Unraveling the aggregation effect on amorphous phase AIE luminogens: a computational study. Nanoscale 2016, 8, 15173-15180.

(19) Zhang, T.; Peng, Q.; Quan, C.; Nie, H.; Niu, Y.; Xie, Y.; Zhao, Z.; Tang, B. Z.; Shuai, Z. Using the isotope effect to probe an aggregation induced emission mechanism: theoretical prediction and experimental validation. Chem. Sci. 2016, 7, 5573-5580.

(20) Zhang, T.; Ma, H.; Niu, Y.; Li, W.; Wang, D.; Peng, Q.; Shuai, Z.; Liang, W. Z. Spectroscopic Signature of the aggregation-induced emission phenomena caused by restricted nonradiative decay: A theoretical proposal. Journal of Physical Chemistry C 2015, 119, 5040-5047.

(21) Zhang, T.; Jiang, Y.; Niu, Y.; Wang, D.; Peng, Q.; Shuai, Z. Aggregation effects on the optical emission of 1,1,2,3,4,5-hexaphenylsilole (HPS): A QM/MM study. Journal of Physical Chemistry A 2014, 118, 9094-9104.

(22) Wu, Q.; Zhang, T.; Peng, Q.; Wang, D.; Shuai, Z. Aggregation induced blue-shifted emission-the molecular picture from a QM/MM study. Physical Chemistry Chemical Physics 2014, 16, 5545-52. 
(23) Wu, Q.; Peng, Q.; Niu, Y.; Gao, X.; Shuai, Z. Theoretical insights into the aggregationinduced emission by hydrogen bonding: A QM/MM study. Journal of Physical Chemistry A 2012, 116, 3881-3888.

(24) Crespo-Otero, R.; Li, Q.; Blancafort, L. Exploring Potential Energy Surfaces for AggregationInduced EmissionFrom Solution to Crystal. Chem. Asian J. 2019, 14, 700-714.

(25) Gierschner, J.; Huang, Y. S.; Van Averbeke, B.; Cornil, J.; Friend, R. H.; Beljonne, D. Excitonic versus electronic couplings in molecular assemblies: The importance of nonnearest neighbor interactions. Journal of Chemical Physics 2009, 130.

(26) Hestand, N. J.; Spano, F. C. Molecular Aggregate Photophysics Beyond the Kasha Model: Novel Design Principles for Organic Materials. Accounts of Chemical Research $2017,50,341-350$.

(27) Shi, J.; Aguilar Suarez, L. E.; Yoon, S. J.; Varghese, S.; Serpa, C.; Park, S. Y.; Lüer, L.; Roca-Sanjuán, D.; Milián-Medina, B.; Gierschner, J. Solid State Luminescence Enhancement in $\pi$-Conjugated Materials: Unraveling the Mechanism beyond the Framework of AIE/AIEE. Journal of Physical Chemistry C 2017, 121, 23166-23183.

(28) Karsili, T. N. V.; Marchetti, B.; Ashfold, M. N. R. Mechanistic Insights into Excited State Intramolecular ProtonTransfer in Isolated and Metal Chelated Supramolecular Chemosensors. Dalton Trans. 2016, 45, 18921-18930.

(29) Azarias, C.; Budzák, .; Laurent, A. D.; Ulrich, G.; Jacquemin, D. Tuning ESIPT Fluorophores into Dual Emitters. Chem. Sci. 2016, 7, 3793-3774.

(30) Ghosh, D.; Ahamed, G.; Batuta, S.; Begum, N. A.; Mandal, D. Effect of an ElectronDonating Substituent at the 3,4-Position of 3-Hydroxyflavone: Photophysics in Bulk Solvents. J. Phys. Chem. A 2016, 120, 44-54. 
(31) Cheng, J.; Liu, D.; Li, W.; Bao, L.; Han, K. Comprehensive Studies on Excited-State Proton Transfer of a Series of 2-(2-Hydroxyphenyl)benzothiazole Derivatives: Synthesis, Optical Properties, and Theoretical Calculations. J. Phys. Chem. C 2015, 119, 42424251.

(32) Baker, L. A.; Horbury, M. D.; Greenough, S. E.; Coulter, P. M.; Karsili, T. N. V.; Roberts, G. M.; Orr-Ewing, A. J.; Ashfold, M. N. R.; Stavros, V. G. Probing the Ultrafast Energy Dissipation Mechanism of the Sunscreen Oxybenzone after UVA Irradiation. J. Phys. Chem. Lett. 2015, 6, 1363-1368.

(33) Zahid, N. I.; Mahmood, M. S.; Subramanian, B.; Mohd Said, S.; Abou-Zied, O. K. New Insight into the Origin of the Red/Near-Infrared Intense Fluorescence of a Crystalline 2-Hydroxychalcone Derivative: A Comprehensive Picture from the Excited-State Femtosecond Dynamics. J. Phys. Chem. Lett. 2017, 5603-5608.

(34) Song, H.; Kuang, Z.; Wang, X.; Guo, Y.; Guo, Q.; Zhang, H.; Xia, A. Solvent Polarity Dependent Excited State Dynamics of 2-Hydroxychalcone Derivatives. J. Phys. Chem. C 2018, 122, 15108-15117.

(35) Shinozaki, Y.; Yamaji, M.; Arai, T. Investigations of excited-state relaxation processes in 2- hydroxychalcone analogues having pyrrole and indole moieties based on emission and transient absorption measurements. J. Photochem. Photobiol., A. 2018, 350, 1722.

(36) Serdiuk, I. E.; Wera, M.; Roshal, A. D. Structural and Spectral Features of 4'Substituted 2'-Hydroxychalcones in Solutions and Crystals: Spectroscopic and Theoretical Investigations. J. Phys. Chem. A 2018, acs.jpca.7b10361.

(37) Li, R.; Yan, L.; Wang, Z.; Qi, Z. An aggregation-induced emissive NIR luminescent based on ESIPT and TICT mechanisms and its application to the detection of Cys. Journal of Molecular Structure 2017, 1136, 1-6. 
(38) Li, J.; Wu, Y.; Xu, Z.; Liao, Q.; Zhang, H.; Zhang, Y.; Xiao, L.; Yao, J.; Fu, H. Tuning the organic microcrystal laser wavelength of ESIPT-active compounds: Via controlling the excited enol and keto emissions. J. Mater. Chem. C 2017, 5, 12235-12240.

(39) Cheng, X.; Li, F.; Han, S.; Zhang, Y.; Jiao, C.; Wei, J.; Ye, K.; Wang, Y.; Zhang, H. Emission behaviors of unsymmetrical 1,3-diaryl- $\beta$-diketones: A model perfectly disclosing the effect of molecular conformation on luminescence of organic solids. Sci. Rep. 2015, 5, 9140 .

(40) Teshima, T.; Takeishi, M.; Arai, T. Red fluorescence from tautomers of 2hydroxychalcones induced by intramolecular hydrogen atom transfer. New J. Chem. 2009, 33, 1393.

(41) Norikane, Y.; Itoh, H.; Arai, T. Photochemistry of 2'-hydroxychalcone. One-way cistrans photoisomerization induced by adiabatic intramolecular hydrogen atom transfer. J. Phys. Chem. A 2002, 106, 2766-2776.

(42) Norikane, Y.; Nakayama, N.; Tamaoki, N.; Arai, T.; Nagashima, U. Quantum Chemical Studies on Photoinduced Cis-Trans Isomerization and Intramolecular Hydrogen Atom Transfer of 2 -Hydroxychalcone. J. Phys. Chem. A 2003, 107, 8659-8664.

(43) Chou, P.-t.; Martinez, M. L.; Cooper, W. C. Direct Evidence of Excited-State Intramolecular Proton Transfer in 2-Hydroxychalcone and Photooxygenation Forming 3-Hydroxyflavone. J. Am. Chem. Soc. 1992, 114, 4943-4944.

(44) Arai, T.; Norikane, Y. Direct Observation of the Photoinduced Hydrogen Atom Transfer in 2'-Hydroxychalcone. 1997.

(45) Jin, X.; Dong, L.; Di, X.; Huang, H.; Liu, J.; Sun, X.; Zhang, X.; Zhu, H. NIR Luminescence for the Detection of Latent Fingerprints Based on ESIPT and AIE Processes. RSC Adv. 2015, 5, 87306-87310. 
(46) Song, Z.; Kwok, R. T. K.; Zhao, E.; He, Z.; Hong, Y.; Lam, J. W. Y.; Liu, B.; Tang, B. Z. A Ratiometric Fluorescent Probe Based on ESIPT and AIE Processes for Alkaline Phosphatase Activity Assay and Visualization in Living Cells. ACS Appl. Mater. Interfaces 2014, 6, 17245-54.

(47) Pil Kim, Y.; Seung Ban, H.; Sung Lim, S.; Kimura, N.; Hoon Jung, S.; Ji, J.; Lee, S.; Ryu, N.; Rok Keum, S.; Hyun Shin, K.; Ohuchi, K. Inhibition of Prostaglandin E 2 Production by 2'-Hydroxychalcone Derivatives and the Mechanism of Action. J. Pharm. Pharmacol 2001, 53, 1295-1302.

(48) Singh, P.; Anand, A.; Kumar, V. Recent Developments in Biological Activities of Chalcones: A Mini Review. Eur. J. Med. Chem. 2014, 85, 758-777.

(49) Cheng, X.; Wang, K.; Huang, S.; Zhang, H.; Zhang, H.; Wang, Y. Organic Crystals with Near-Infrared Amplified Spontaneous Emissions Based on 2-Hydroxychalcone Derivatives: Subtle Structure Modification but Great Property Change. Angew. Chem., Int. Ed. 2015, 54, 8369-8373.

(50) Tang, B.; Liu, H.; Li, F.; Wang, Y.; Zhang, H. Single-Benzene Solid Emitters with Lasing Properties Based on Aggregation-Induced Emissions. Chem. Commun. 2016, 52, 6577-6580.

(51) Cheng, X.; Zhang, Y.; Han, S.; Li, F.; Zhang, H.; Wang, Y. Multicolor Amplified Spontaneous Emissions Based on Organic Polymorphs That Undergo Excited-State Intramolecular Proton Transfer. Chem. Eur. J. 2016, 22, 4899-4903.

(52) Dommett, M.; Crespo-Otero, R. Excited Etate Proton Transfer in 2-Hydroxychalcone Derivatives. Phys. Chem. Chem. Phys. 2017, 19, 2409-2416.

(53) Dommett, M.; Rivera, M.; Crespo-Otero, R. How Inter- and Intramolecular Processes Dictate Aggregation-Induced Emission in Crystals Undergoing Excited-State Proton Transfer. J. Phys. Chem. Lett. 2017, 8, 6148-6153. 
(54) Martinez, C. R.; Iverson, B. L. Rethinking the term "pi-stacking". Chem. Sci. 2012, 3, 2191-2201.

(55) Aragó, J.; Troisi, A. Dynamics of the Excitonic Coupling in Organic Crystals. Phys. Rev. Lett. 2015, 114, 1-5.

(56) Fornari, R. P.; Aragó, J.; Troisi, A. Exciton Dynamics in Phthalocyanine Molecular Crystals. J. Phys. Chem. C 2016, 120, 7987-7996.

(57) Aragó, J.; Troisi, A. Regimes of Exciton Transport in Molecular Crystals in the Presence of Dynamic Disorder. Adv. Funct. Mater. 2016, 26, 2316-2325.

(58) Stehr, V.; Fink, R. F.; Engels, B.; Pflaum, J.; Deibel, C. Singlet Exciton Diffusion in Organic Crystals Based on Marcus Transfer Rates. J. Chem. Theory Comput. 2014, $10,1242-1255$.

(59) Kimura, A.; Kakitani, T.; Yamato, T. Theory of Excitation Energy Transfer in the Intermediate Coupling Case. II. Criterion for Intermediate Coupling Excitation Energy Transfer Mechanism and Application to the Photosynthetic Antenna System. The Journal of Physical Chemistry B 2000, 104, 9276-9287.

(60) Barbatti, M.; Ruckenbauer, M.; Plasser, F.; Pittner, J.; Granucci, G.; Persico, M.; Lischka, H. Newton-X: A surface-hopping program for nonadiabatic molecular dynamics. Wiley Interdiscip. Rev. Comput. Mol. Sci. 2014, 4, 26-33.

(61) Crespo-Otero, R.; Barbatti, M. Spectrum simulation and decomposition with nuclear ensemble: Formal derivation and application to benzene, furan and 2-phenylfuran. Theor. Chem. Acc. 2012, 131, 1-14.

(62) Niu, Y.; Peng, Q.; Deng, C.; Gao, X.; Shuai, Z. Theory of Excited State Decays and Optical Spectra: Application to Polyatomic Molecules. J. Phys. Chem. A 2010, 114, $7817-7831$. 
(63) Giannozzi, P. et al. QUANTUM ESPRESSO: A Modular and Open-Source Software Project for Quantum Simulations of Materials. J. Phys. Condens. Matter 2009, 21, 395502 .

(64) Campbell, J. E.; Yang, J.; Day, G. M. Predicted EnergyStructureFunction Maps for the Evaluation of Small Molecule Organic Semiconductors. J. Mater. Chem. C 2017, $5,7574-7584$.

(65) Rappé, A. K.; Bormann-Rochotte, L. M.; Wiser, D. C.; Hart, J. R.; Pietsch, M. A.; Casewit, C. J.; Skiff, W. M. APT a Next Generation QM-Based Reactive Force Field Model. Mol. Phys. 2007, 105, 301-324.

(66) Reimers, J. R. A Practical Method for the Use of Curvilinear Coordinates in Calculations of Normal-Mode-Projected Displacements and Duschinsky Rotation Matrices for Large Molecules. J. Chem. Phys. 2001, 115, 9103-9109.

(67) Levine, B. G.; Coe, J. D.; Martínez, T. J. Optimizing Conical Intersections Without Derivative Coupling Vectors: Application to Multistate Multireference Second-Order Perturbation Theory (MS-CASPT2). J. Phys. Chem. B 2008, 112, 405-413.

(68) Crespo-Otero, R.; Kungwan, N.; Barbatti, M. Stepwise Double Excited-State Proton Transfer is Not Possible in 7-Azaindole Dimer. Chem. Sci. 2015, 6, 5762-5767.

(69) Frisch, M. J. et al. Gaussian 09, Revision D.01. 2009.

(70) Aquilante, F. et al. Molcas 8: New Capabilities for Multiconfigurational Quantum Chemical Calculations Across the Periodic Table. J. Comput. Chem. 2016, 37, 506541.

(71) Rivera, M.; Dommett, M.; Crespo-Otero, R. ONIOM(QM:QM) Electrostatic Embedding Schemes for Photochemistry in Molecular Crystals. J. Chem. Theory Comput. 2019, 15, 2504-2516. 


\section{Supporting Information For:}

\section{Molecular and Crystalline Requirements for Solid}

State Fluorescence Exploiting Excited State

\section{Intramolecular Proton Transfer}

Michael Dommett, Miguel Rivera, Matthew T. H. Smith, Rachel Crespo-Otero*

School of Biological and Chemical Sciences, Materials Research Institute,

Queen Mary University of London, Mile End Road, London E1 4NS, UK

E-mail: r.crespo-otero@qmul.ac.uk 


\section{Contents}

$\begin{array}{ll}\text { S1 Crystal Packing Properties } & 3\end{array}$

$\begin{array}{lr}\text { S2 Huang-Rhys Factors } & 8\end{array}$

$\begin{array}{lr}\text { S3 Electronic excitations } & 12\end{array}$

S4 Exciton Coupling in Trimers $\quad 14$

$\begin{array}{lr}\text { S5 H- and J-Aggregates } & 16\end{array}$

$\begin{array}{lr}\text { S6 Bias for ESIPT } & 19\end{array}$

$\begin{array}{lr}\text { S7 Orbitals in CASPT2 Calculations } & 20\end{array}$

S8 Crystalline Emission Spectra $\quad 21$

$\begin{array}{lr}\text { References } & 21\end{array}$ 


\section{S1 Crystal Packing Properties}
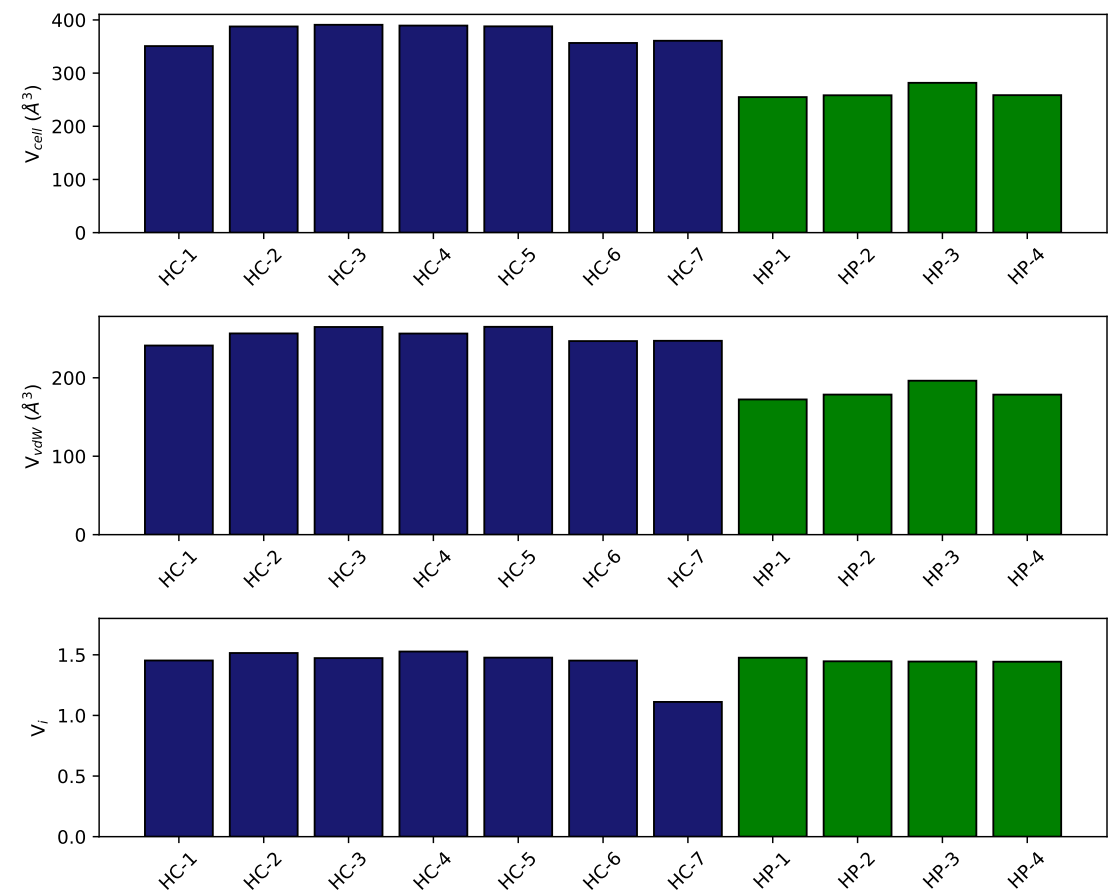

Figure S1: Voronoi cell volumes $\left(\mathrm{V}_{\text {cell }}\right)$, van der Waals volumes $\left(\mathrm{V}_{\mathrm{vdW}}\right)$ and the index $\left(\mathrm{V}_{i}=\mathrm{V}_{\text {cell }} / \mathrm{V}_{\mathrm{vdW}}\right)$ 


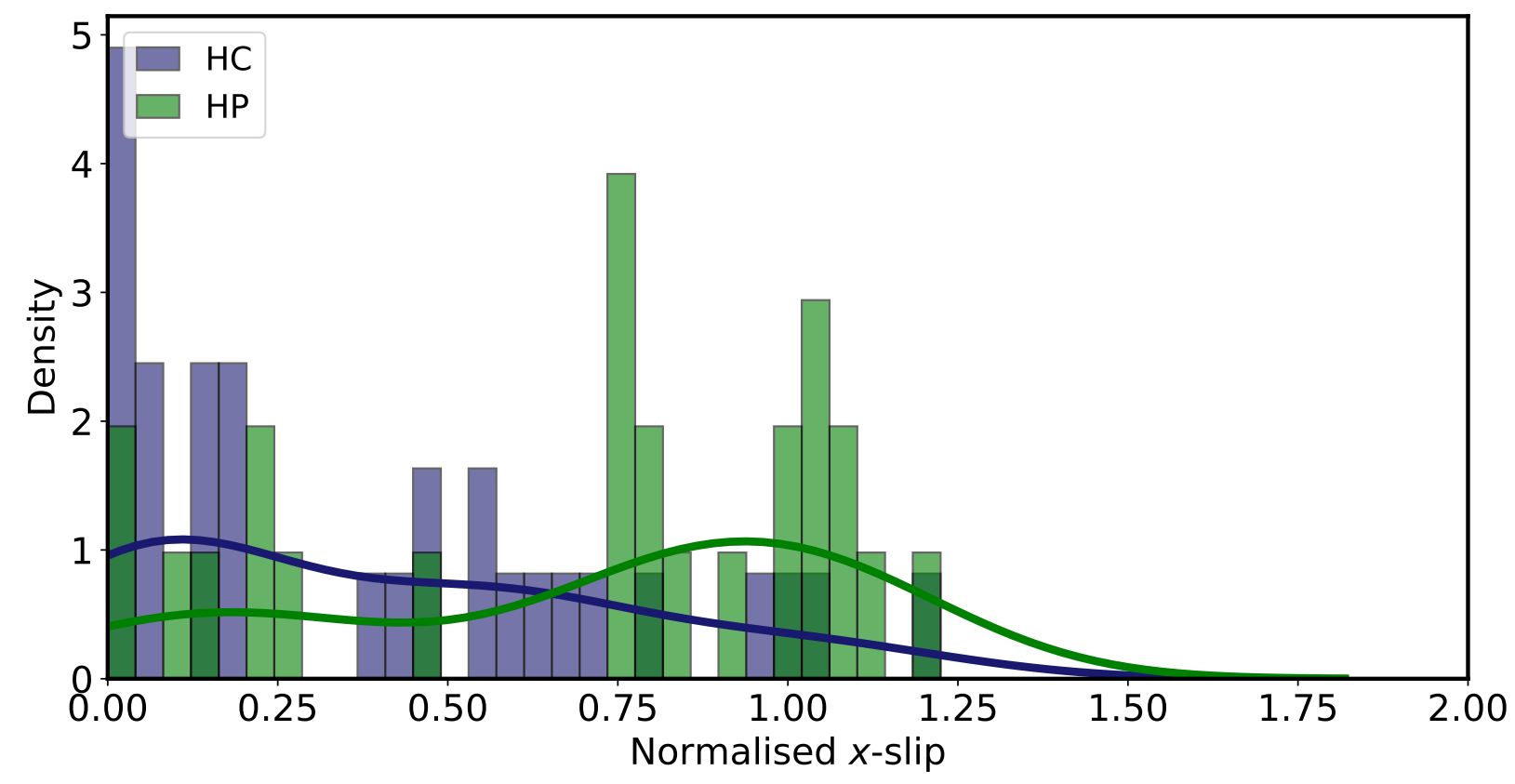

Figure S2: The density of slip distances for each molecular dimer in the $x$-plane (long axis) for cofacial dimers. The slip is normalised by the length of the long axis $x$ for each molecule, such that slip is molecule independent. 

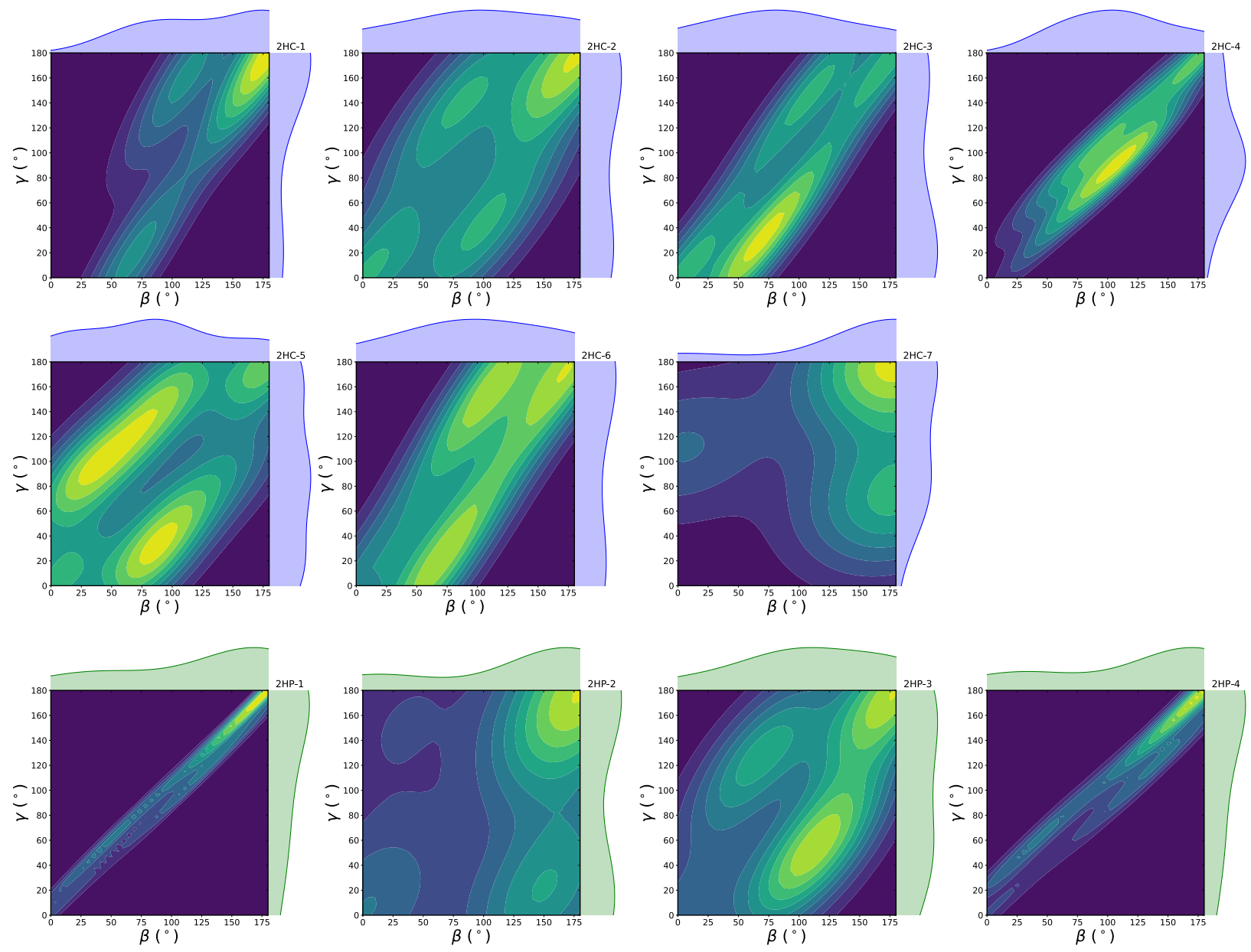

Figure S3: Probability density map of $\beta$ and $\gamma$ angles for each of the systems under study 
Table S1: Dimer classification definitions

\begin{tabular}{cccc}
\hline & $\alpha$ Condition & $\beta$ Condition & $\gamma$ Condition \\
\hline Herringbone & $\alpha \leq 60^{\circ}$ & $45^{\circ} \leq \beta \leq 135^{\circ}$ & $\gamma \leq 45^{\circ} \vee\left(135^{\circ} \leq \gamma \leq 225^{\circ}\right)$ \\
Parallel T & $\alpha \leq 60^{\circ}$ & $\beta \leq 45^{\circ} \vee\left(135^{\circ} \leq \beta \leq 225\right)$ & $45^{\circ} \leq \gamma \leq 135^{\circ}$ \\
Perpendicular T & $\alpha \leq 60^{\circ}$ & $45^{\circ} \leq \beta \leq 135^{\circ}$ & $45^{\circ} \leq \gamma \leq 135^{\circ}$ \\
CoFacial & $\alpha \leq 60^{\circ}$ & $\beta \leq 45^{\circ} \vee\left(135^{\circ} \leq \beta \leq 225\right)$ & $\gamma \leq 45^{\circ} \vee\left(135^{\circ} \leq \gamma \leq 225^{\circ}\right)$ \\
\hline
\end{tabular}

Table S2: Dimer classifications in each molecular crystal for $\alpha \leq 60^{\circ}$. Significant increase in HC5 dimers due to rotational flexibility of the methoxy group.

\begin{tabular}{ccccc}
\hline System & Herringbone & Parallel-T & Perpendicular-T & CoFacial \\
\hline HC1 & 3 & 0 & 0 & 1 \\
HC2 & 4 & 0 & 0 & 0 \\
HC3 & 3 & 0 & 0 & 0 \\
HC4 & 1 & 0 & 4 & 0 \\
HC5 & 9 & 6 & 4 & 3 \\
HC6 & 3 & 0 & 0 & 1 \\
HC7 & 0 & 3 & 0 & 0 \\
\hline HP1 & 0 & 0 & 3 & 1 \\
HP2 & 0 & 0 & 0 & 2 \\
HP3 & 0 & 0 & 2 & 1 \\
HP4 & 0 & 3 & 0 & 1 \\
\hline
\end{tabular}


Herringbone Parallel

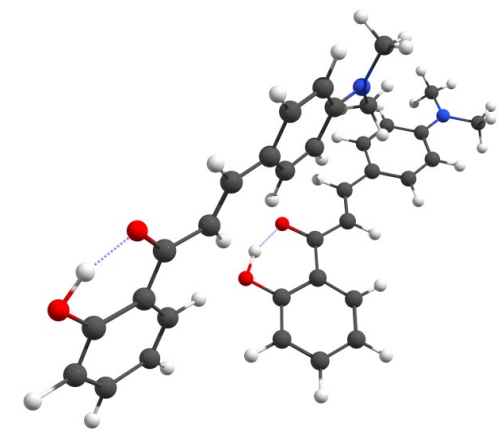

$\alpha: 56^{\circ} \quad \beta: 68^{\circ} \quad \gamma: 18^{\circ}$

CoFacial Parallel

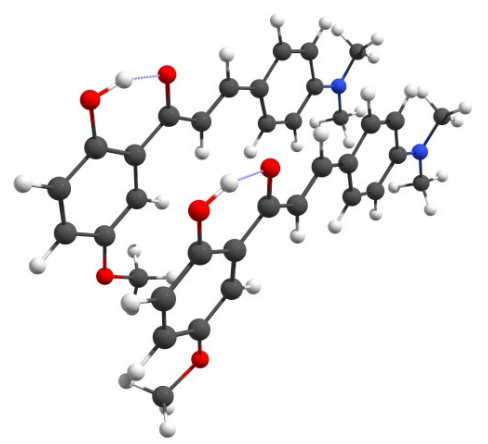

$\alpha: 16^{\circ} \quad \beta: 5^{\circ} \quad \gamma: 3^{\circ}$

Parallel T

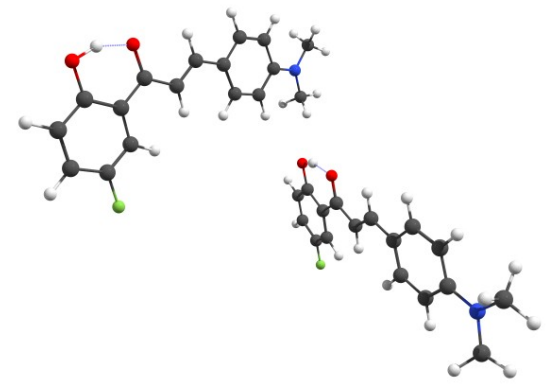

$\alpha: 41^{\circ} \quad \beta: 3^{\circ} \quad \gamma: 111^{\circ}$
Herringbone Antiparallel

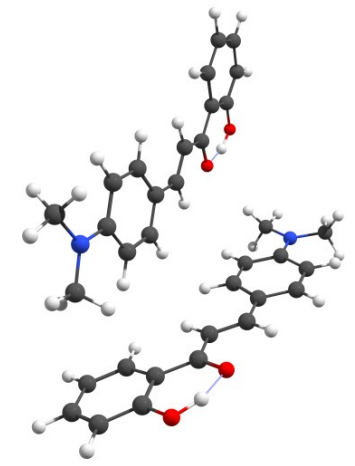

$\alpha: 18^{\circ} \quad \beta: 112^{\circ} \quad \gamma: 162^{\circ}$

CoFacial-Antiparallel

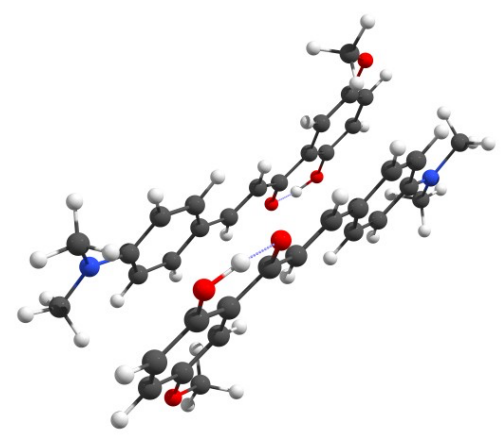

$\alpha: 11^{\circ} \quad \beta: 180^{\circ} \gamma: 180^{\circ}$

Perpendicular T

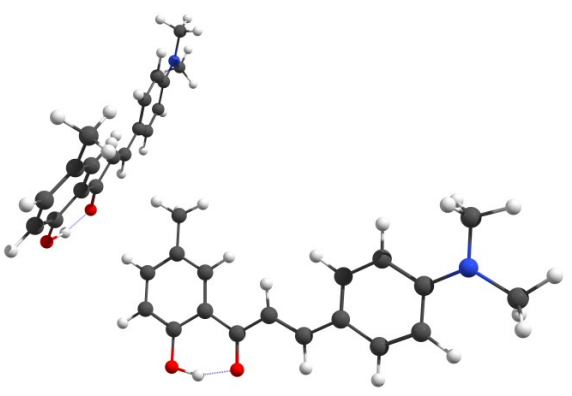

$\alpha: 22^{\circ} \quad \beta: 58^{\circ} \quad \gamma: 80^{\circ}$

Figure S4: Example dimer packing modes and associated $\alpha, \beta$ and $\gamma$ angles in HC1, HC5, and HP1. 


\section{S2 Huang-Rhys Factors}

Normal modes can be correlated between different electronic states using the the Duschinsky rotation matrix, yielding the Huang-Rhys factors $S$ between the electronic states for each frequency $\omega$

$$
S_{j}=\frac{\omega_{j} D_{j}^{2}}{2 \hbar}
$$

where $D_{j}$ is the displacement of mode $j$ between the equilibrium geometry in the considered electronic states. Summation of each Huang-Rhys factor yields the normal mode reorganisation energy for the system

$$
\lambda_{N M}=\sum_{j=1}^{3 N-6} \hbar \omega_{j} S_{j}
$$

In this section, we calculate the HR factors for HC1, HC5, and HP1.

The Huang-Rhys (HR) factors in vacuum and molecular crystals were calculated for $\mathbf{H C 1}$,

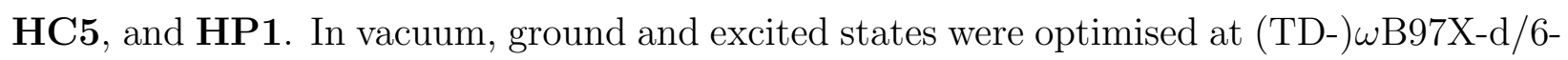
$31 \mathrm{G}(\mathrm{d})$ level. In the molecular crystal, a cluster model consisting of a central chromophore and all molecules within a $7 \AA$, taken from the optimised unit cell for each system (see main text for unit cell optimisation details). Ground and excited states were optimised at

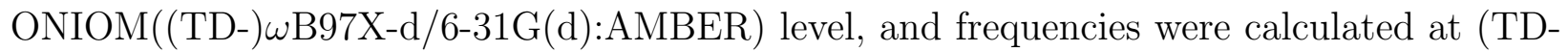
)wB97X-d/6-31G(d) level using point charge embedding. The DUSHIN program was used to calculate the Huang-Rhys factors and the associated reorganisation energies $\left(\lambda_{N M}\right)$.

Figures S5-S7 show the Huang-Rhys (HR) factors in vacuum and solid state for HC1, HC5 and HP1. The different y-axis scales for the Huang-Rhys factors between plots should be noted. Each system is discussed in turn below. 

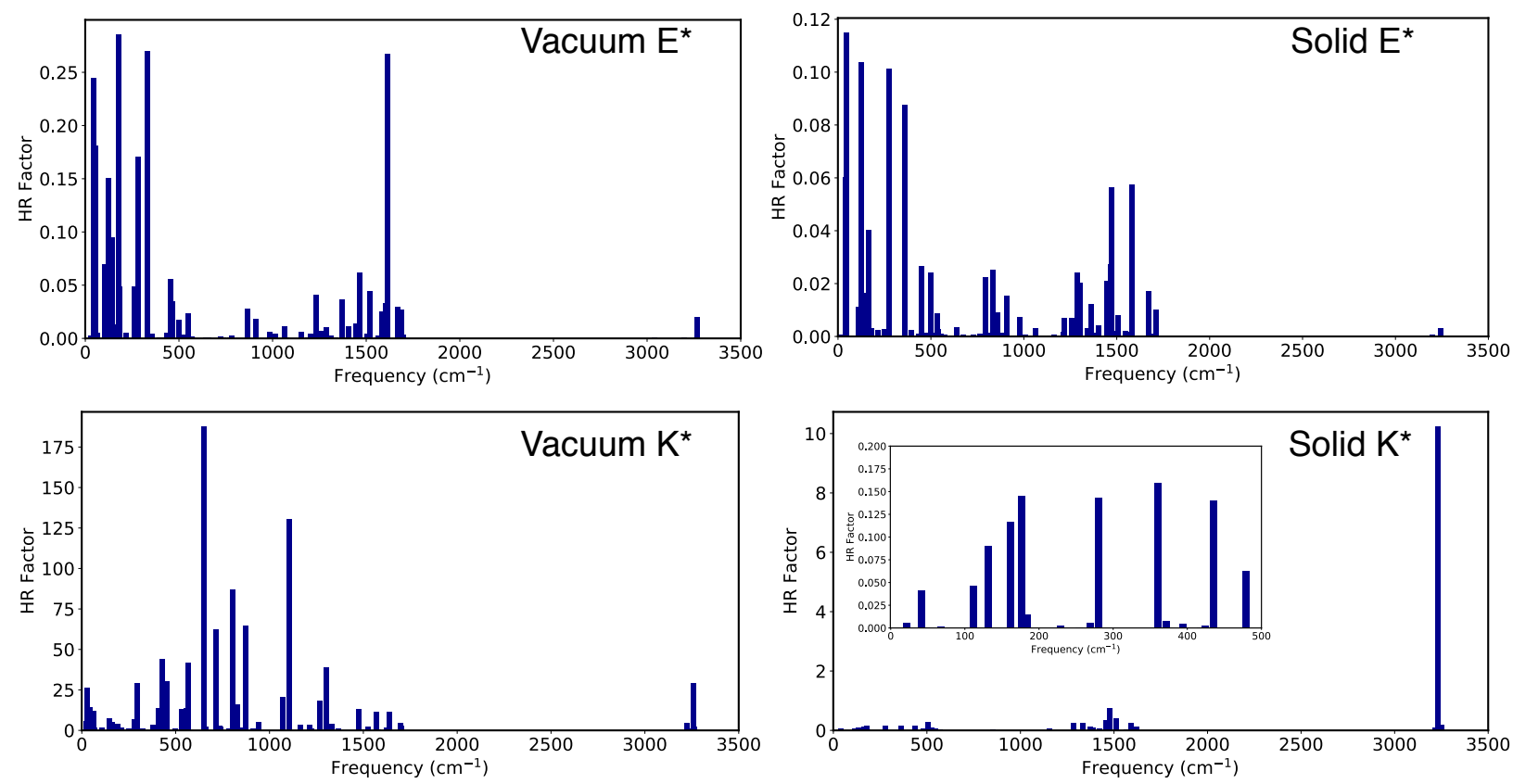

Figure S5: Huang-Rhys factors associated with each normal mode calculated via the Duschinsky rotation matrix between the $\mathrm{E}^{*}$ and $\mathrm{S}_{0}$, and $\mathrm{K}^{*}$ and $\mathrm{S}_{0}$ electronic states for HC1. Frequencies $0-500 \mathrm{~cm}^{-1}$ in the solid state are shown in the inset.
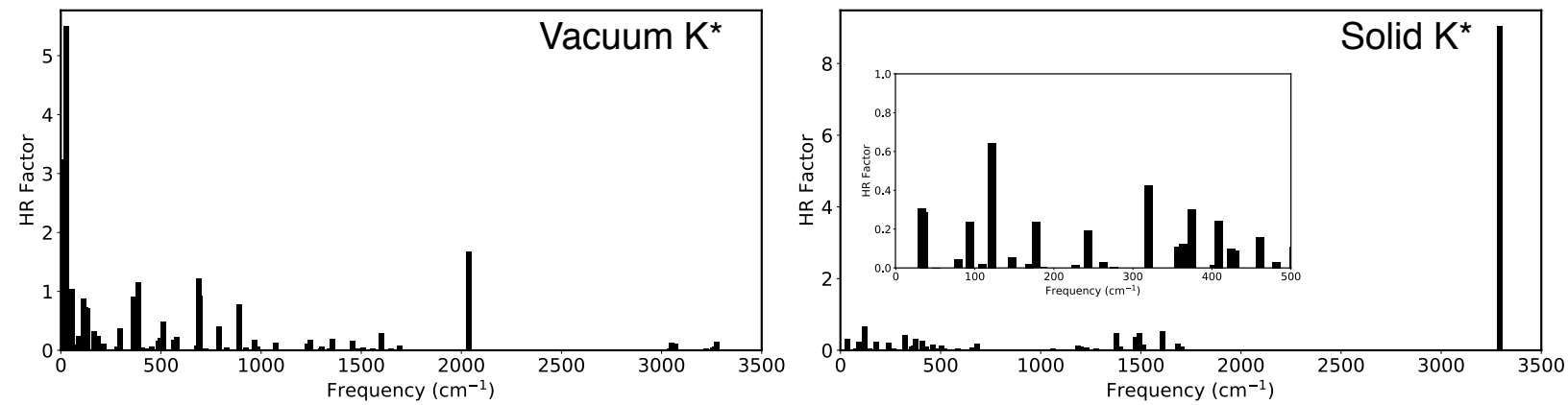

Figure S6: Huang-Rhys factors associated with each normal mode calculated via the Duschinsky rotation matrix between $\mathrm{K}^{*}$ and $\mathrm{S}_{0}$ electronic states for HC5. Frequencies 0-500 $\mathrm{cm}^{-1}$ in the solid state are shown in the inset. 

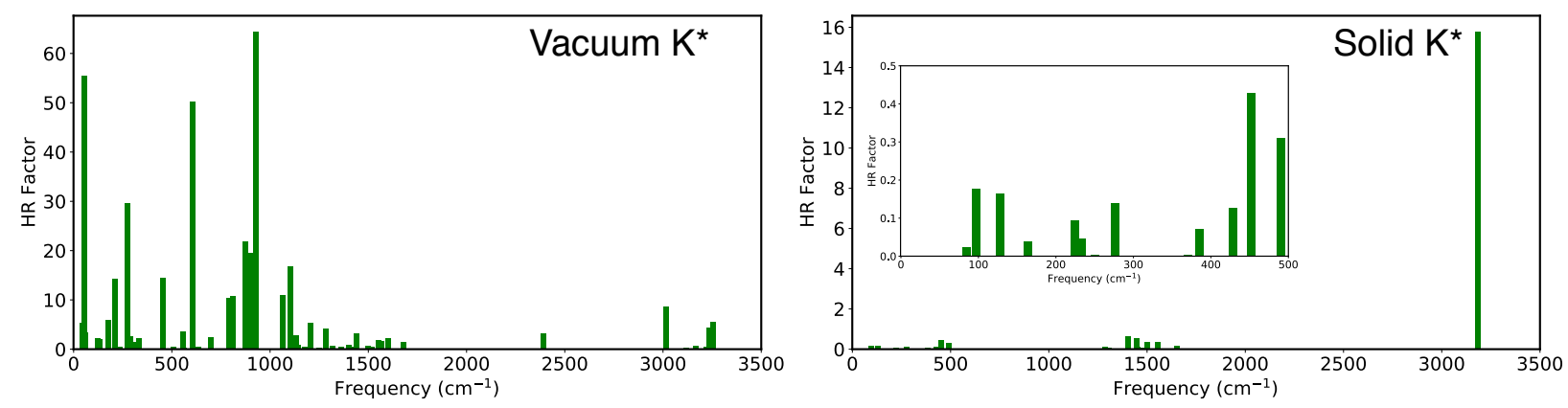

Figure S7: Huang-Rhys factors associated with each normal mode calculated via the Duschinsky rotation matrix between $\mathrm{K}^{*}$ and $\mathrm{S}_{0}$ electronic states for $\mathbf{H P 1}$. Frequencies $0-500 \mathrm{~cm}^{-1}$ in the solid state are shown in the inset.

For $\mathrm{HC1}$ in vacuum (Figure S5, left), the geometric similarity between the planar E* excited state minimum and the ground state equilibrium geometry yields negligible HR factors. This leads to $\lambda_{N M}$ of $0.08 \mathrm{eV}$, which underestimates the $\lambda_{A}$ of 0.36 . For $\mathrm{K}^{*}$, the PES in vacuum is highly anharmonic, and intramolecular rotation leads to a highly distorted geometry with respect to the ground state. As a consequence, the HR factors are extremely large and the harmonic approximation is non-applicable for determining the reorganisation energy, as shown by a value of $99 \mathrm{eV}\left(\lambda_{A}=3.64 \mathrm{eV}\right)$. Moving to the molecular crystal (Figure $\mathrm{S} 5$, right), in $\mathrm{E}^{*}$ the $\mathrm{HR}$ factors are of similar magnitude as in vacuum but in $\mathrm{K}^{*}$ they are markedly reduced and suppressed to fractional values. The largest $\mathrm{K}^{*} \mathrm{HR}$ factor is the $\mathrm{O}-\mathrm{H}$ stretching mode, since the geometry remains planar and has the largest displacement between $\mathrm{S}_{0}$ and $\mathrm{S}_{1}$ as it is the ESIPT coordinate. The $\mathrm{K}^{*} \lambda_{N M}$ is $4.67 \mathrm{eV}$, with $\lambda_{A}=0.59 \mathrm{eV}$.

In HC5 there is no stable $\mathrm{E}^{*}$ minimum in either vacuum or the solid state for the monomer chromophore. In vacuum, the HR factors are much less than in $\mathbf{H C 1}$ due to the rotation angle at the minimum being less distorted. The molecule is more planar and is closer in structure and vibrational signature. At larger rotation angles the MECI is reached (see Section S6). HR factors are reduced in the solid state, in particular for the rotational modes, but with the $\mathrm{O}-\mathrm{H}$ stretch $\mathrm{HR}$ factor increasing.

For HP1, large reorganisation energies, and correspondingly large HR-factors, are associated with low-frequency rotational modes. Indeed, such is the displacement between 
modes in the ground and excited states, the total reorganisation energy is $41 \mathrm{eV}$, whereas the adiabatic value $3.89 \mathrm{eV}$. As such, the harmonic approximation is invalid here due to the excited state potential energy surface anharmonicity. In the solid state, the normal modes associated with rotation are significantly reduced, whilst the largest HR factor is associated with the stretching of the phenol oxygen. This is to be expected, since it is along this coordinate that ESIPT occurs, and hence is has the largest HR-factor. In solid state, $\lambda_{N M}$ is $2.19 \mathrm{eV}$, compared with $\lambda_{A}$ of $1.24 \mathrm{eV}$.

In the case of the $\mathbf{H C}$ and $\mathbf{H P}$ systems, the AIE behaviour can not be directly attributed to the reduction of the HR factors in the solid state compared to vacuum. Such is the complexity of the PES, where ESIPT and rotation occur in the excited state (in vacuum), the surfaces are highly anharmonic and the validity of the HR scheme via Duschinsky is not clear. As such, it is not the focus of our investigation into the AIE behaviour of these systems.

Table S3: Reorganisation energies $(\mathrm{eV})$ in the adiabatic regime for each system in the enol (where located) and keto minima

\begin{tabular}{ccc}
\hline System & $\mathrm{E}^{*}$ & $\mathrm{~K}^{*}$ \\
\hline HC1 & 0.233 & 0.786 \\
HC2 & 0.246 & 0.804 \\
HC3 & 0.249 & 0.668 \\
HC4 & 0.250 & 0.935 \\
HC5 & - & 0.978 \\
HC6 & 0.210 & 0.533 \\
HC7 & 0.213 & 0.649 \\
\hline HP1 & - & 1.238 \\
HP2 & - & 1.059 \\
HP3 & - & 1.120 \\
HP4 & - & 1.204 \\
\hline
\end{tabular}




\section{S3 Electronic excitations}

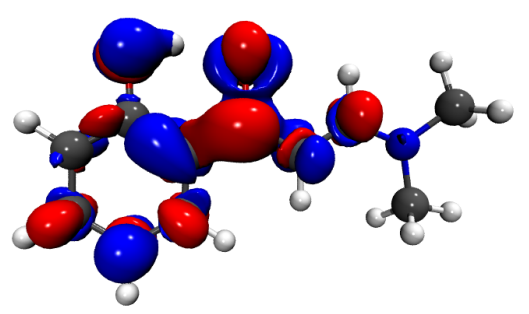

$\mathrm{S}_{1}$

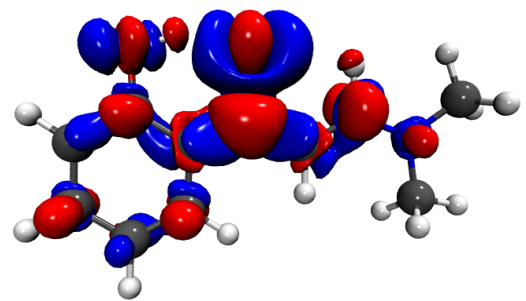

$\mathrm{S}_{2}$

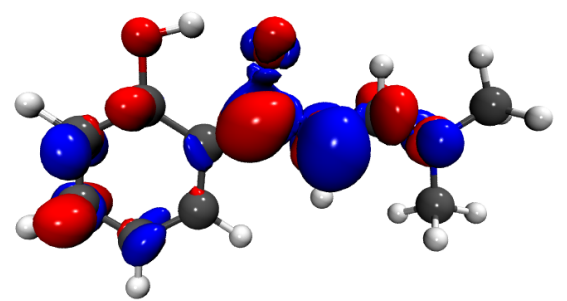

$\mathrm{S}_{3}$

Figure S8: Electron density difference maps for the first three excitations of HP1. Blue regions represent electron density loss from the ground state and red represent electron density gain in the excited state, with isovalue of 0.002. Calculated at TD- $\omega$ B97X-D/6$311++\mathrm{G}(\mathrm{d}, \mathrm{p})$ in vacuum. 

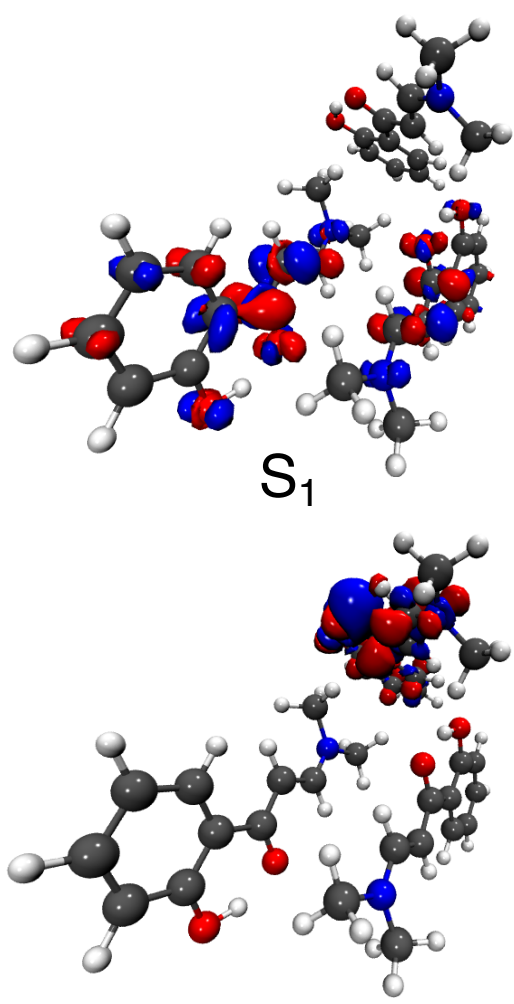

$\mathrm{S}_{4}$

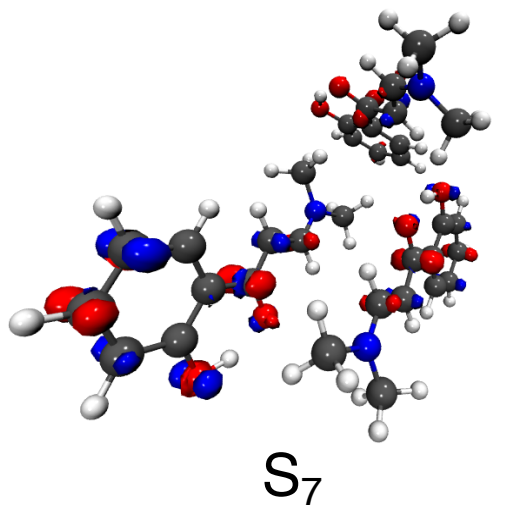

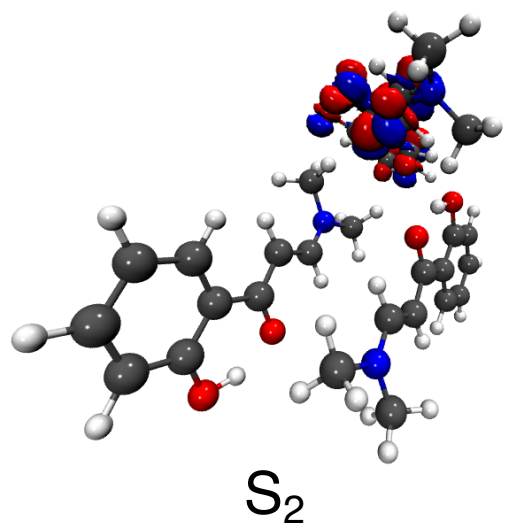
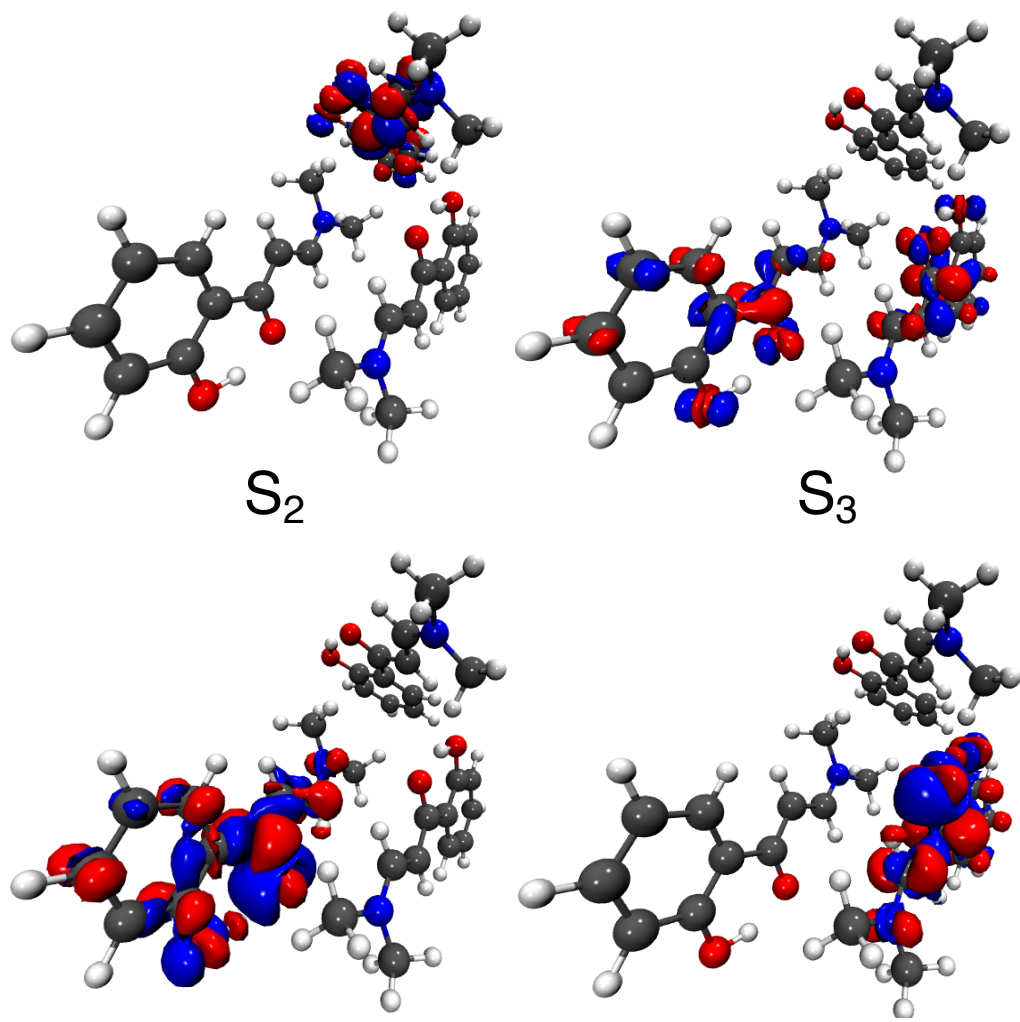

$\mathrm{S}_{5}$

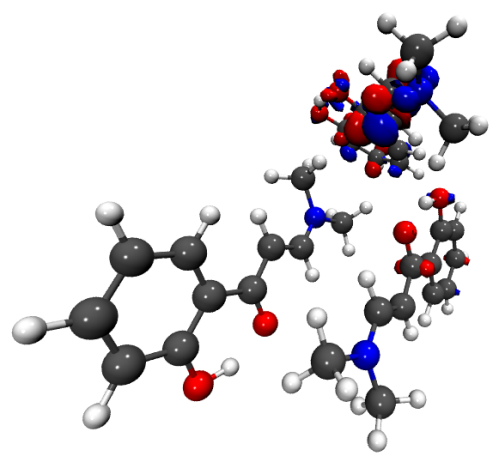

$\mathrm{S}_{8}$
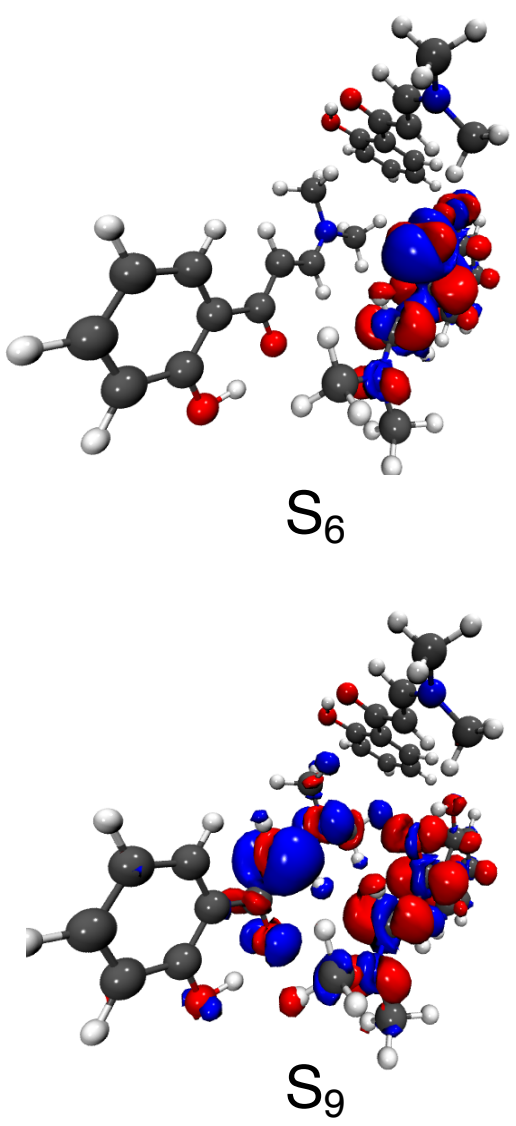

Figure S9: Electron density diffrence maps for the first nine excited state of the HP1 trimer. The same colour scheme is used as in Figure S8 


\section{S4 Exciton Coupling in Trimers}

The exciton coupling in trimers was calculated for HC1, HC5, and HP1. The results are given in Table S4, and shown schematically in Figure S9, where the couplings obtained in a dimer scheme are compared to those obtained when an additional molecule is included.

It is found that the addition of a third molecule has only a small effect on the dimer coupling in HP1 and HC1, where the increased coupling in one dimer is compensated for by the decreased coupling in the other dimer, with difference of less that $0.02 \mathrm{eV}$. The largest effect is seen in HC5 due to the cofacial packing of the trimer system, where the central monomer is sandwiched by two cofacially stacked monomers, one parallel and one antiparallel. In this system, the parallel dimer $(\gamma=0)$ has its coupling increased by $0.04 \mathrm{eV}$ with the addition of the third molecule, which is aligned antiparallel $(\gamma=180)$. Conversely, the antiparallel coupling is reduced by $0.07 \mathrm{eV}$ due to the parallel stacked monomer.

Table S4: Exciton coupling comparison for dimer and trimer in $\mathbf{H C 1}$, HC5, and HP1

\begin{tabular}{|c|c|c|}
\hline System & Dimer Coupling (eV) & Trimer Coupling (eV) \\
\hline \multirow{3}{*}{ HC1 } & 0.108 & 0.090 \\
\hline & 0.108 & 0.125 \\
\hline & 0.061 & 0.063 \\
\hline \multirow{3}{*}{ HC5 } & 0.061 & 0.052 \\
\hline & $\overline{0} . \overline{1} \overline{1} \bar{C}^{-}$ & $\overline{0} . \overline{1} \overline{5} \overline{7}$ \\
\hline & 0.142 & 0.085 \\
\hline \multirow{3}{*}{ HP1 } & $-\overline{0} . \overline{0} \overline{4}$ & $\overline{0} \overline{0} \overline{0} \overline{1}$ \\
\hline & 0.056 & 0.066 \\
\hline & 0.005 & 0.014 \\
\hline
\end{tabular}



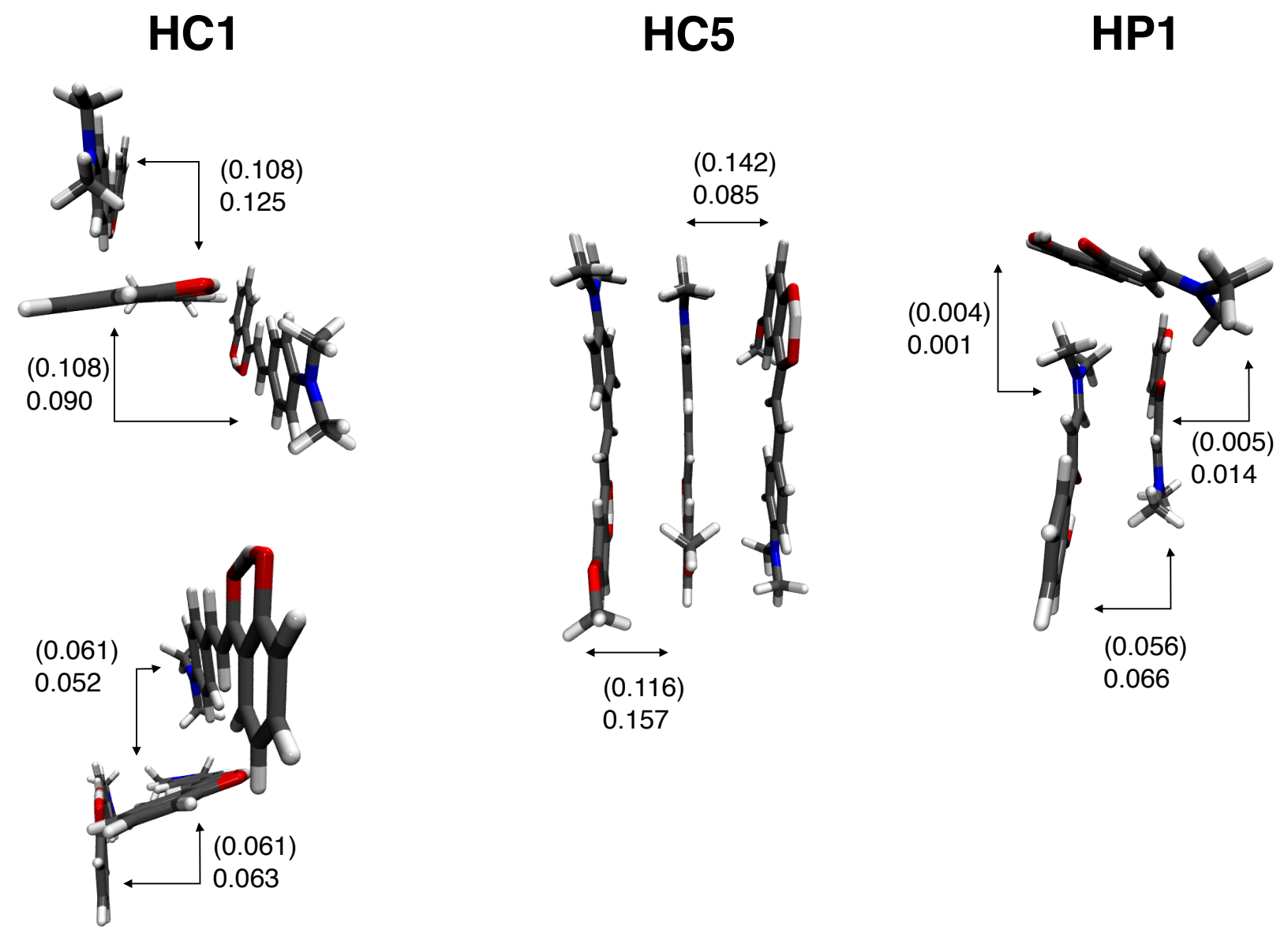

Figure S10: Schematic of the trimer motifs extracted from unit cells of HC1, HC5 and HP1. Exciton couplings for dimer pairs (shown in brackets) and then for the full trimer are shown. Calculated at $\omega \mathrm{B} 97 \mathrm{X}-\mathrm{D} / 6-311++\mathrm{G}(\mathrm{d}, \mathrm{p})$ level of theory using the diabatization described in the main text. 


\section{S5 H- and J-Aggregates}
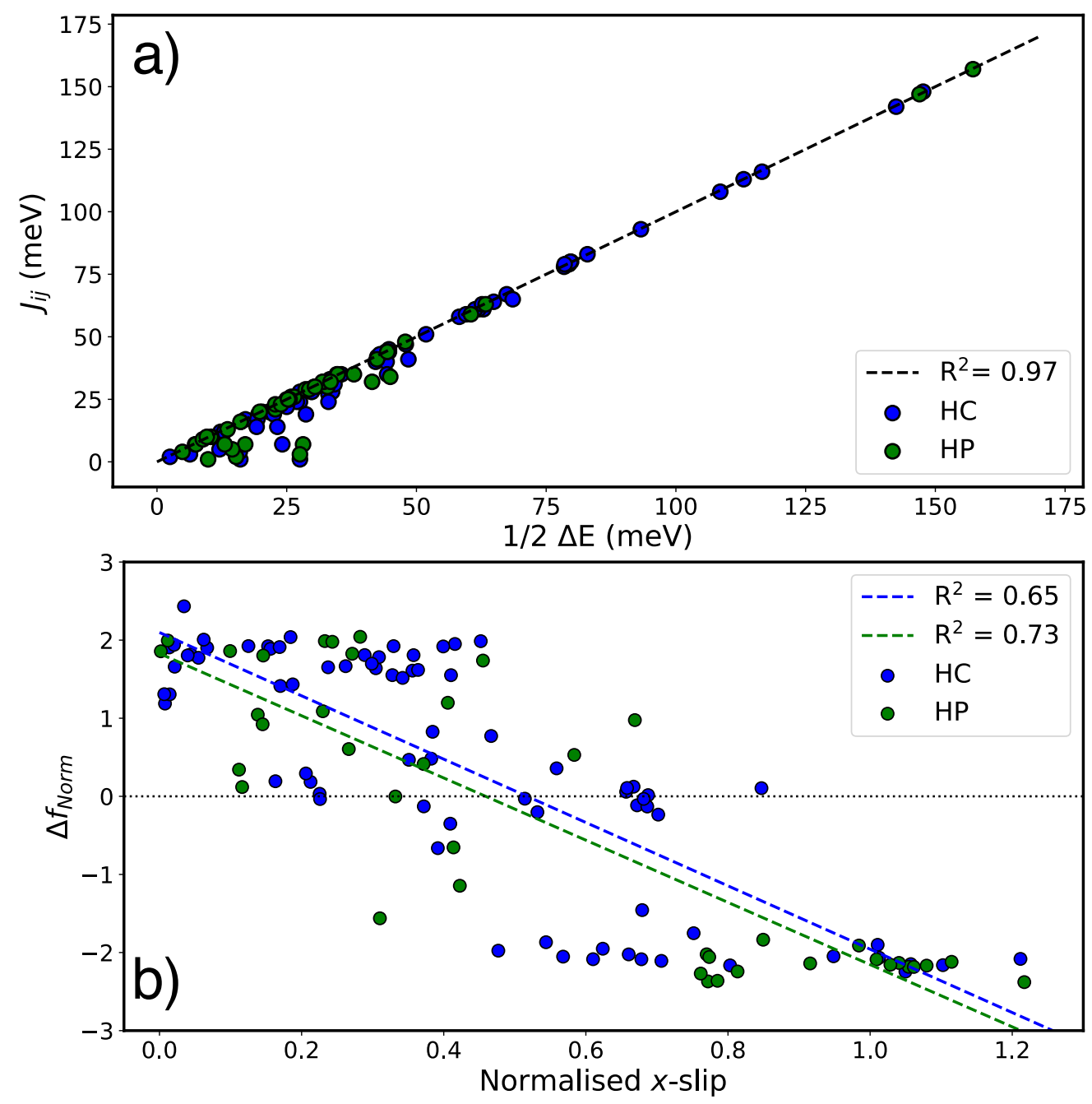

Figure S11: Panel a), top; Correlation between the energy splitting of the dimer states and the diabatic exciton coupling. Panel b), bottom; Linear regression of the $x$-slip against the difference in oscillator strength between the $S_{2}$ and $S_{1}$ states in dimers. The definition of $\Delta f_{N o r m}$ is given in Equation S3

As shown in Figure S11a, the coupling $J$ correlates linearly with half of the energy splitting for the $\mathrm{S}_{1}$ and $\mathrm{S}_{2}$ states of the dimer. The energy splitting is perhaps the simplest way to obtain the exciton coupling in the Kasha regime, although it is more expensive than using atomic-centred transition charges, or the PDA approximation, since the supermolecular calculation must be done rather than one monomer calculation. At small intermolecular distances $(<4 \AA)$, these computationally efficient metrics can underestimate the couplings 
due to them only considering the Coulomb interaction. ${ }^{1}$ The linear correlation here shows that the general Kasha interpretation of the coupling applies here and that the diabatization method to obtain the couplings reproduces the supramolecular coupling.

Table S5: Dimer types located for each molecular crystal. Significant increase in HC5 dimers due to rotational flexibility of the methoxy group

\begin{tabular}{cccc}
\hline System & H-aggregates & J-aggregates & Total \\
\hline HC1 & 4 & 4 & 8 \\
HC2 & 5 & 4 & 9 \\
HC3 & 4 & 5 & 9 \\
HC4 & 7 & 2 & 9 \\
HC5 & 19 & 10 & 29 \\
HC6 & 4 & 3 & 7 \\
HC7 & 6 & 0 & 6 \\
\hline HP1 & 5 & 5 & 10 \\
HP2 & 5 & 6 & 11 \\
HP3 & 4 & 5 & 9 \\
HP4 & 5 & 5 & 10 \\
\hline
\end{tabular}

In the Kasha model, for a perfectly stacked dimer with no $x$-slip, the oscillator strength of the $\mathrm{S}_{2}$ state should be double that of the monomer state. Figure S11b shows the relationship between the $x$-slip in the dimers and the oscillator strength, namely the difference in oscillator strength between the $\mathrm{S}_{2}$ and $\mathrm{S}_{1}$ states in the dimer, normalised by the corresponding mononomer oscillator strength

$$
\Delta f_{\text {Norm }}=\frac{f_{S_{2}}^{\text {dimer }}-f_{S_{1}}^{\text {dimer }}}{f_{S_{1}}^{\text {monomer }}} .
$$

These systems generally fit the Kasha model, as when the $x$-slip is zero, the line of best fit predicts an enhanced $\mathrm{S}_{2}$ intensity of 2.10 for the $\mathbf{H C s}$ and 1.83 for the HPs. With increasing $x$-slip, the difference in oscillator strength between the two states decreases until the inversion to J-aggregates is witnessed $\left(f_{S_{1}}>f_{S_{2}}\right)$. For the $\mathbf{H C}$ s this occurs at a $x$-slip of $52 \%$ and at $46 \%$ for the HCs. However, there is some signficant noise in the model, which is understandable given the simplicity of the model and the complex electronic structure of the dimer. The largest group of outliers are cofacially stacked dimers, where a larger shift is 
seen at lower slip distances due to the minimal $x$-slip and archetypal stacking. ${ }^{2}$ 


\section{S6 Bias for ESIPT}

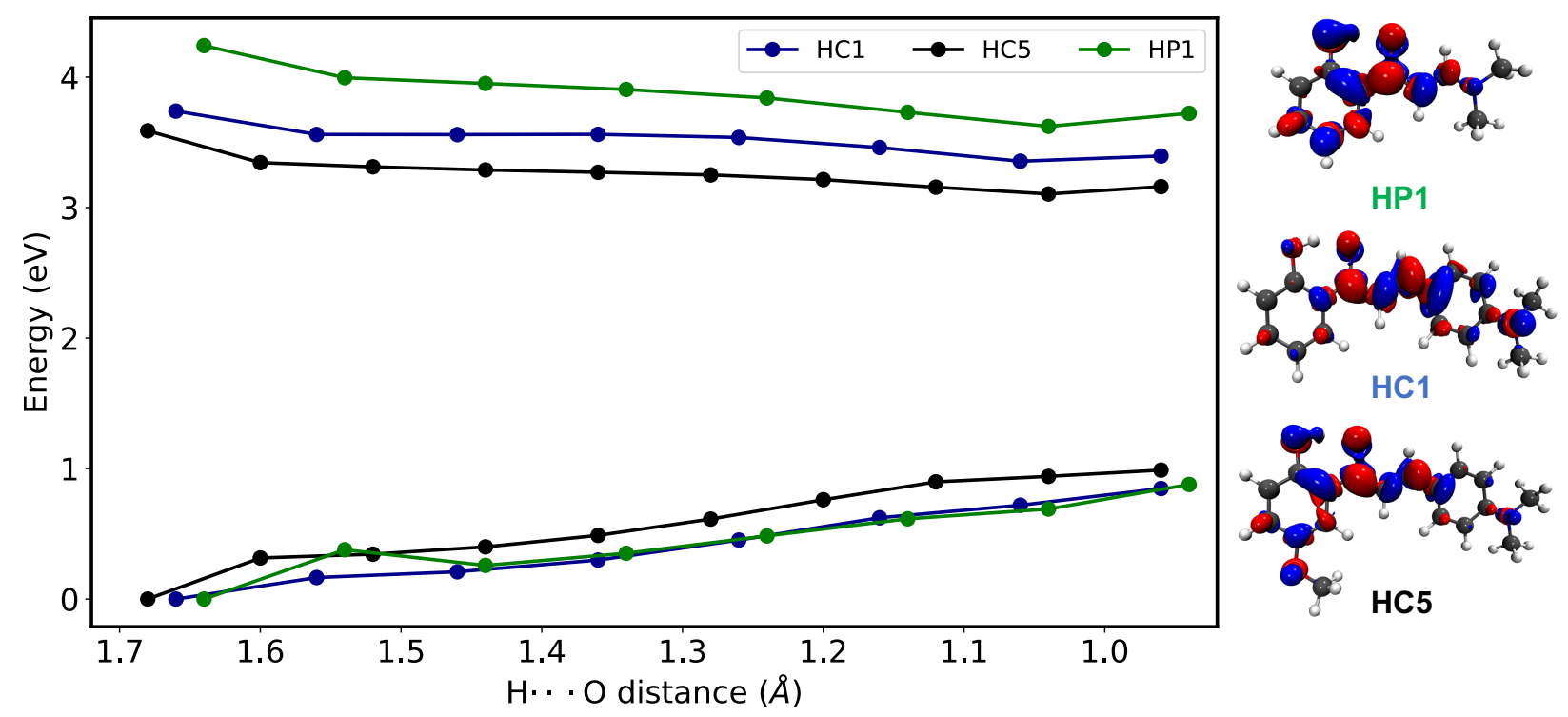

Figure S12: Relaxed geometry scan of the phenol hydrogen to carbonyl oxygen distance for HC1, HC5 and HP1, calculated at TD- $\omega$ B87X-D/6-31G(d). Electron density difference maps are also shown, with same labelling as for Figure S8.

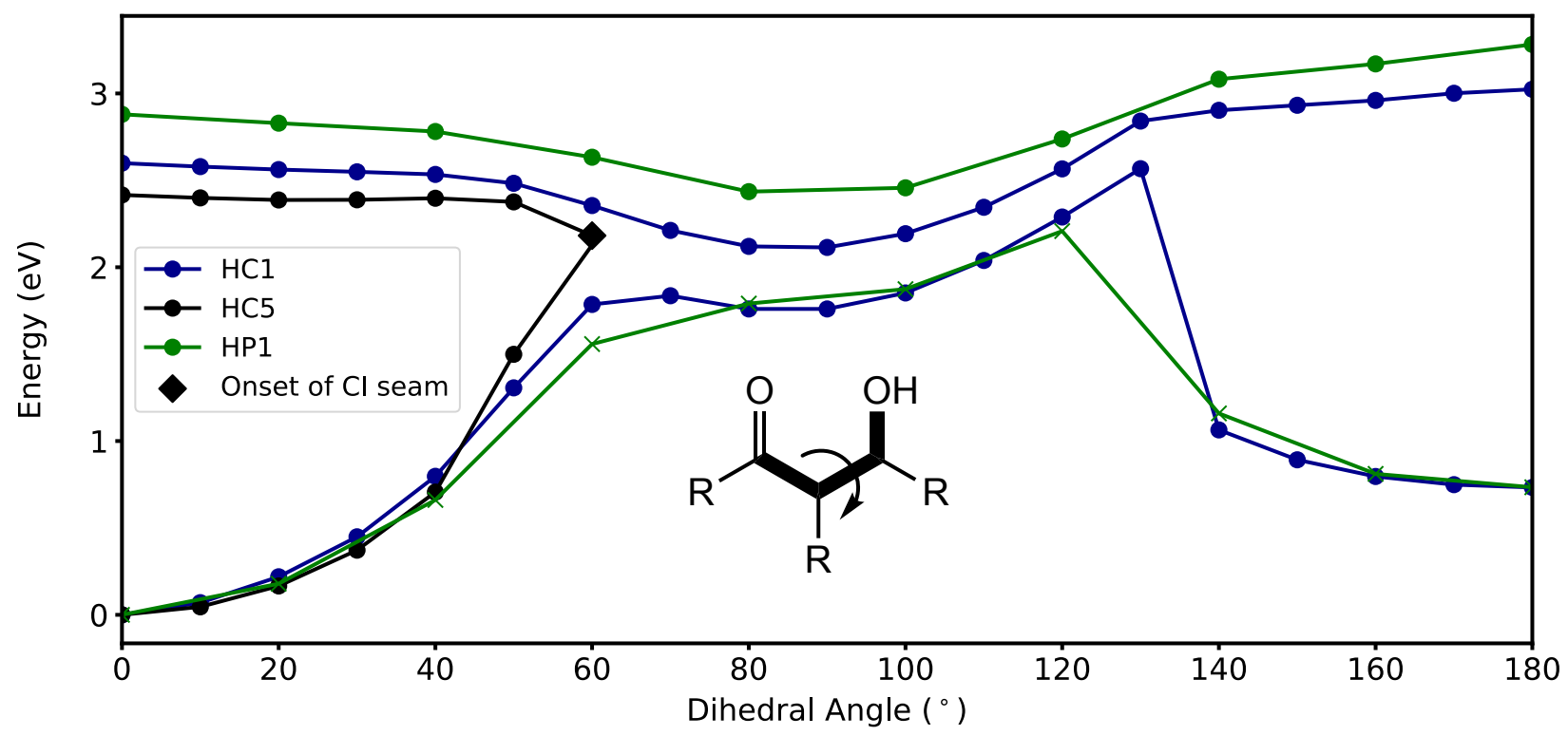

Figure S13: Relaxed geometry scan of the torsional angle (shown inset) for HC1, HC5 and HP1 in vacuum, calculated at TD- $\omega$ B87X-D/6-31G(d). For HC5, the scan cannot proceed further than $60^{\circ}$ due to the convergence of the two electronic states. 


\section{S7 Orbitals in CASPT2 Calculations}

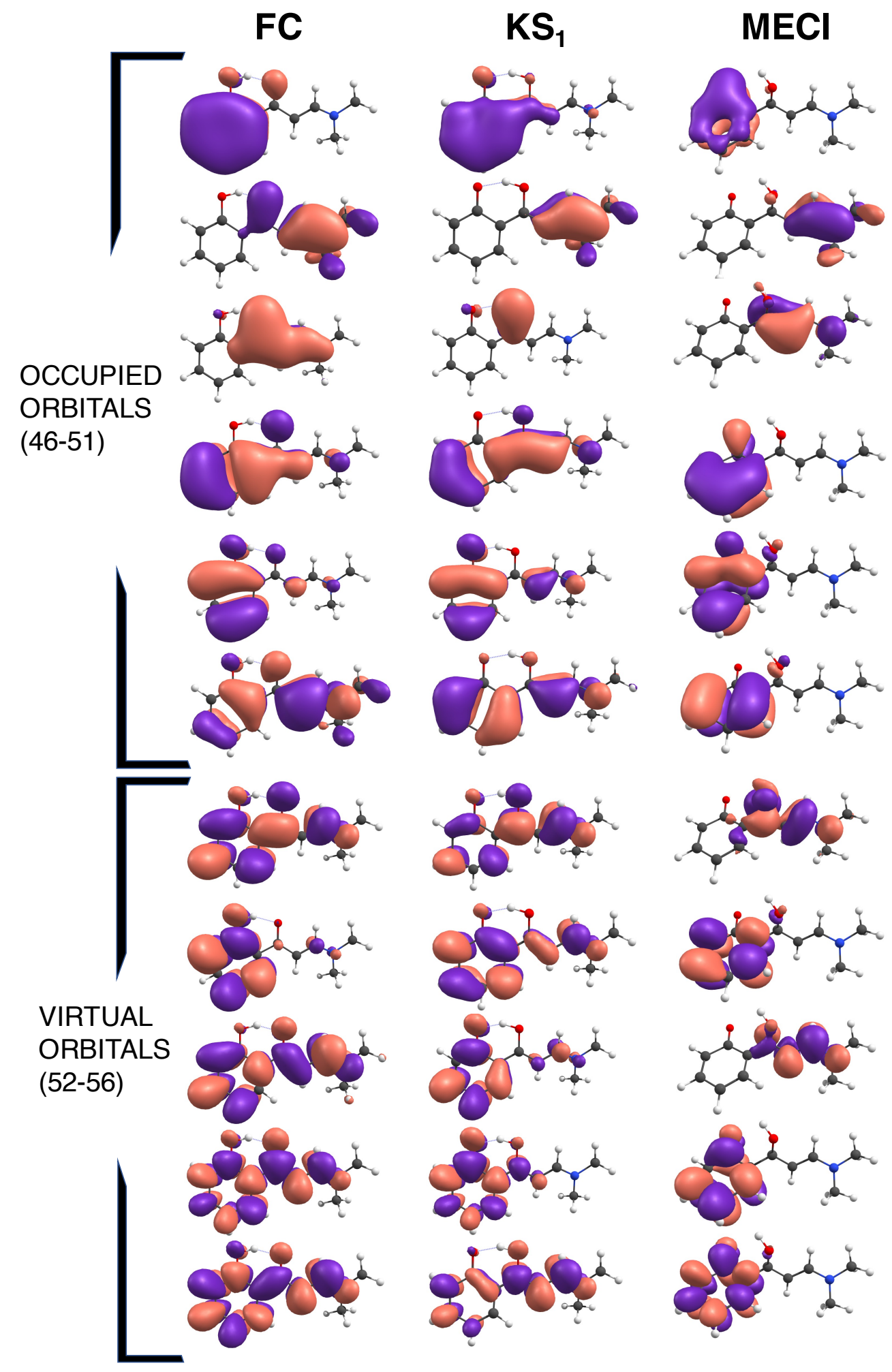

Figure S14: Orbitals in the active space for the CASPT2 calculations, where the active space consists of 12 electrons in 11 orbitals. Orbitals shown for each of the Franck-Condon (FC), keto $\mathrm{S}_{1}\left(\mathrm{KS}_{1}\right)$ and minimum energy conical intersection (MECI) calculations. 


\section{S8 Crystalline Emission Spectra}

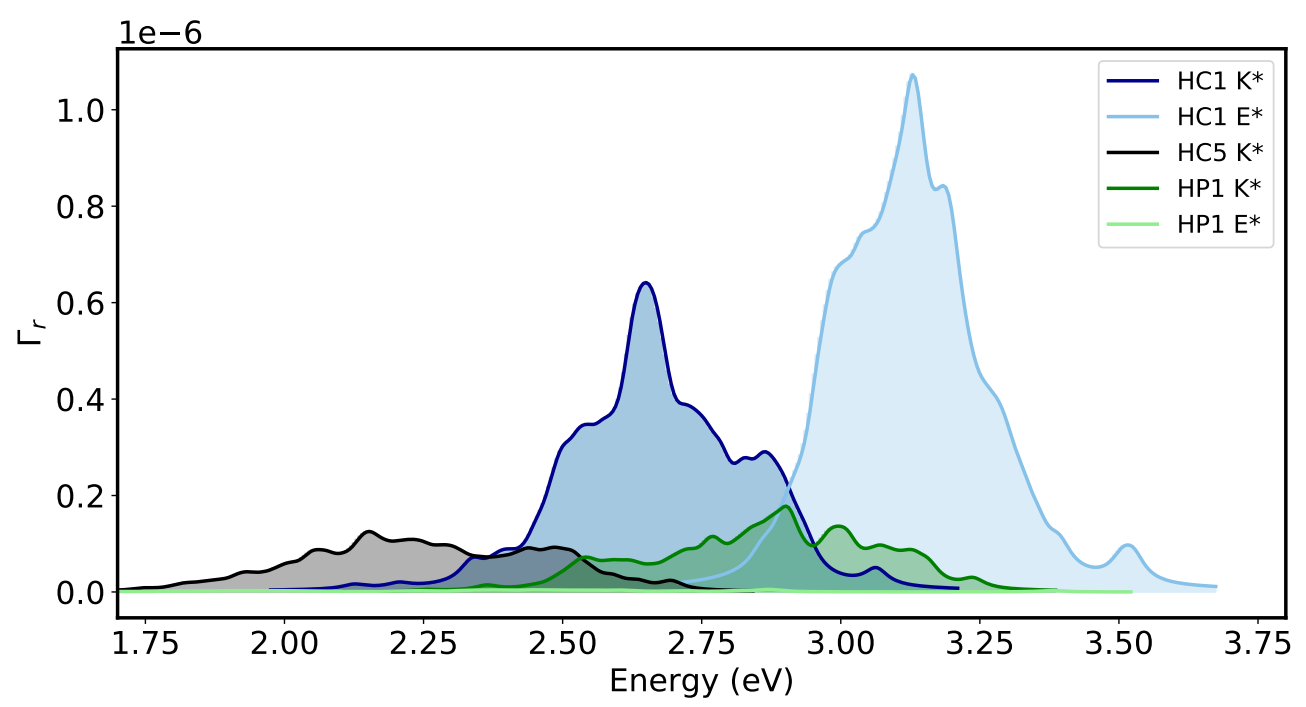

Figure S15: Emission spectra in molecular crystal for $7 \AA$ clusters for HC1, HC5 and HP1, calculated at TD- $\omega$ B97X-D/6-31G(d) in point charges. Single point energies were calculated for 100 initial conditions based upon a Wigner distribution of the excited state frequencies calculated at ONIOM(TD- $\omega$ B97X-D/6-31G(d):AMBER) level.

\section{References}

(1) Kistler, K. A.; Spano, F. C.; Matsika, S. A benchmark of excitonic couplings derived from atomic transition charges. Journal of Physical Chemistry B 2013, 117, 2032-2044.

(2) Gierschner, J.; Varghese, S.; Park, S. Y. Organic Single Crystal Lasers: A Materials View. Advanced Optical Materials 2016, 4, 348-364. 
